\title{
LA FRONTIÈRE COMME ENJEU LES ANNALES ET LA SOCIOLOGIE
}

\author{
Jérôme LAmY et Arnaud SAINT-Martin*
}

RÉsumÉ: Les Annales ont tracé, depuis leurs débuts dans les années 1930, des frontières avec la sociologie. L'étude de trois moments majeurs (l'ère des fondateurs, le moment Braudel et la décennie 1980) permet de saisir le travail d'horographie comme un enjeu sans cesse actualisé dans les rapports de force institutionnels et épistémologiques. L'identité collective des Annales, émergeant dans ces débats de délimitation avec la sociologie, s'appuie sur un réseau de valeurs et de références continuellement ajusté.

MotS-CLÉs: frontières, discipline, revue.

\section{BOUNDARIES AT STAKE. THE ANNALES SCHOOL AND SOCIOLOGY}

ABSTRACT: Since their beginnings in the 1930's, the Annales periodically demarcated themselves with sociology. Focusing on three decisive moments (the foundation, the Braudel moment, and the eighties), this article documents the process of boundaries drawing, which is unvaryingly at stakes in the context of institutional and epistemological disputes. The collective identity of the Annales resulted from the definition of continuously adjusted values.

KEYWORDS: boundaries, discipline, journal.

* Jérôme Lamy, né en 1976, est post-doctorant à l'université de Strasbourg. Ses recherches portent sur l'émergence d'une compétence spatiale en France après le second conflit mondial ainsi que sur la constitution de la sismologie comme savoir mondialisé. II a publié L'Observatoire de Toulouse aux XVIII ${ }^{e}$ et XIX siècles. Archéologie d'un espace savant (Rennes, Presses universitaires de Rennes, 2007).

Adresse: Institut de recherches interdisciplinaires sur les sciences et la technologie, Bât. le SET, 7, rue de 1'Université, F-67000 Strasbourg.

Courrier électronique: jerome.lamy@laposte.net

Arnaud Saint-Martin, né en 1979, a soutenu en 2008 à l'université de Paris-Sorbonne une thèse intitulée L'Office et le télescope. Une sociologie historique de l'astronomie française, 1900-1940. Ses recherches contribuent à l'élaboration d'une sociologie transversaliste des sciences. Ses derniers travaux portent sur la psychiatrie contemporaine et plus spécifiquement sur l'émergence de la catégorie de « trouble de la personnalité ».

Adresse: École des hautes études en sciences sociales, Centre de sociologie européenne, 54, boulevard Raspail, F-75006 Paris.

Courrier électronique: arsaint-martin@orange.fr 
L es rapports entre l'histoire et la sociologie ne laissent pas de susciter d'innombrables débats depuis la fin du XIX ${ }^{\mathrm{e}}$ siècle. En France, les controverses récurrentes émaillant l'avènement de la sociologie - durkheimienne, cela va sans dire - sont riches de malentendus persistants, de positions intellectuelles souvent crispées, d'appropriations ambivalentes. Les territoires couverts par les deux disciplines sont régulièrement marqués à l'occasion d'épreuves critiques qui enjoignent historiens et sociologues à justifier leur positionnement épistémologique. Même les points de vue les plus manifestement détachés des intérêts en jeu - se revendiquant de l'épistémologie des sciences sociales, par exemple ${ }^{1}-$ ne manquent pas d'engager une conception normative, ne serait-ce qu'en ce qui concerne les modalités suivant lesquelles il conviendrait de traiter le problème (ou le faux problème, c'est selon). Affirmer, par exemple, que la différence entre histoire et sociologie est une « affaire de discipline ${ }^{2}$ », un artifice intellectuel expliqué par des différends professionnels ${ }^{3}$ ou bien encore qu'elle résulte de la pluralisation de «formes discursives » irréductibles ${ }^{4}$, c'est immanquablement laisser entrevoir un jugement de valeur. En sorte que chaque contribution au débat, quels que soient finalement le degré de sa réflexivité, de sa fortune et de son éventuelle valeur cognitive, est une pièce de plus à verser dans les archives.

Cet article propose d'analyser les luttes de définition des territoires disciplinaires et les mobilisations intellectuelles à l'œuvre dans l'interprétation du clivage entre histoire et sociologie. Il met en lumière des processus d'horographie ${ }^{5}$, c'est-à-dire des pratiques de traçage de frontières inséparablement intellectuelle et institutionnelle. Il montre en particulier comment celles-ci sont travaillées à l'échelle d'une revue (les Annales) et d'une discipline (la sociologie, un temps symbolisée par L'Année sociologique). Cette dissymétrie apparente constitue un choix méthodologique visant à ne pas ramener la question des frontières interdisciplinaires uniquement à un affrontement bipolaire entre disciplines déjà constituées dans (et à travers) leur différence. Il ne suffit pas de saisir la frontière dans l'ordre des savoirs à travers les découpages ex post d'aires cohérentes et stables en fonction de repères épistémologiques et institutionnels, pour attester ensuite de leurs écarts éventuels. Encore faut-il restituer la dynamique des pratiques de démarcation symbolique, leurs mouvements et leur évolution à diverses échelles et dimensions et sur différentes scènes ${ }^{6}$.

Les Annales sont l'espace d'expression d'une intention disciplinaire, c'est-à-dire de la volonté, collectivement orchestrée, d'organiser un champ intellectuel selon des normes particulières. C'est le discours sur l'histoire que ses fondateurs entendent monopoliser. La revue est fondée en 1929 par Marc Bloch et Lucien Febvre dans le

1. REVEL, 2001.

2. Berthelot, 1998.

3. Passeron, 1991, ici 2006, p. 125-168.

4. HaLl, 1999.

5. Nous empruntons librement le terme au géopoliticien Michel Foucher. Formé à partir de la racine grecque « horoi », le néologisme renvoie aux limites politiques du territoire des cités (FoUCHER, 1991, p. 49).

6. Les « pratiques de démarcation » (boundary-work) sont un terrain de choix de la cultural sociology anglo-américaine comme des études sociales des sciences. La littérature est désormais abondante. Nous renvoyons, notamment, à CEFAÏ, 2007, p. 467-545; EvANS, 2005 ; GIERYN, 1995; LAMONT et Molnar, 2002. 
but de promouvoir une certaine façon de faire l'histoire. Les deux historiens impulsent un style particulier aux Annales. Le ton des recensions est « direct et massif, débarrassé de fioritures ${ }^{7} »$; la revue participe d'une politique d'affirmation (travaillant l'identité de la revue) en même temps que d'une stratégie de type ubiquiste (imposant la variété des approches). Depuis la Faculté des Lettres de l'université de Strasbourg - terrain excentré d'expérimentation interdisciplinaire ${ }^{8}$-, les Annales protègent le champ en établissant une " veille aux frontières ${ }^{9} »$ : l'espace de recherche trusté par la revue est affirmé et défendu contre d'éventuelles tentatives de redéfinition endogènes et exogènes. Les Annales tirent ainsi leur légitimité de l'institutionnalisation d'une " coupure épistémologique ${ }^{10} »$; c'est un collectif dont l'ambition est de fournir un ensemble d'outils - empruntés notamment à d'autres disciplines que l'histoire - pour servir des fins épistémiques et professionnelles propres. Ni strictement une « école », ni véritablement une discipline à elles seules, les Annales brouillent les repères, résistent aux étiquetages de toutes sortes ${ }^{11}$ et se placent à l'avant-poste (frontière) des sciences sociales sans jamais renier l'identité de leur histoire ${ }^{12}$.

Les Annales offrent un point d'observation privilégié pour appréhender des césures cognitives et institutionnelles. En effet, la revue a, dès sa création, intégré la question des identités et des marqueurs disciplinaires comme principe fondateur. Toutefois la frontière ne sera envisagée dans cet article que d'un côté (i.e. celui des Annales) afin de ne pas étendre l'analyse au-delà du raisonnable. Il n'en reste pas moins qu'une semblable enquête sur les processus de délimitation cognitive pourrait être menée à propos d'autres revues. Le recoupement de ces travaux devrait mettre au jour l'évolution des découpages dans l'ordre des savoirs, les tensions institutionnelles qu'ils génèrent ainsi que les transformations des rapports de force entre disciplines, revues et éditeurs. Notre but n'est pas de reconstituer une nouvelle fois l'histoire des Annales; l'historiographie est, sur ce point, surabondante. L'objet saisi dans cette étude est donc moins une surface éditoriale particulière qu'un objet historiographique, la « frontière », dont la polysémie est pour nous particulièrement heuristique. L'horographie des territoires socio-intellectuels

7. Burguière, 2006, p. 26.

8. Craig, 1984, p. 231.

9. Pour reprendre une expression de FABIANI, 1988, p. 97.

10. Fabiani, 2006, p. 20.

11. Fernand Braudel est lui-même rétif à ces labellisations au nom d'une indépendance dans la recherche: «[...] j'ai le sentiment que, quand il s'agit de l'équipe des Annales, du mouvement, ou de la pseudo-école, des Annales, ce qu'on lui reproche, c'est d'avoir ce souci particulier d'une liberté indispensable, qui est liberté de l'esprit de recherche, quelle que soit la découverte, si découverte il y a, que nous rencontrerons » (BRAUDEL, 1997, p. 147). Sur les processus rétrospectifs d'étiquetage scientifique et en particulier sur l'usage stratégique de la notion d' « école », voir Topalov, 2004.

12. Le lien peut d'ailleurs être fait avec l'histoire d'autres processus d'institutionnalisation. Nous pensons par exemple à la mise en forme progressive de l'American Journal of Sociology, telle que la reconstitue Andrew Abbott dans Department and Discipline. Le périodique est l'instrument de publicité d'un département universitaire qui parvient à élaborer une certaine image de la sociologie, fondée sur des manières de faire, de savoir et d'envisager la profession. Mieux, explique Abbott, l'American Journal of Sociology contribue dans ses trente premières années à créer la discipline labélisée « sociologie ». Avec d'autres institutions, il lui donne l'assise éditoriale sans laquelle la disciplinarisation n'opère que partiellement. En ce sens, il est un marqueur symbolique et un support matériel de disciplinarité, au même titre que L'Année sociologique et les Annales. Voir Aввотт, 1999. 
constitue un dispositif conceptuel quasi expérimental que nous initions et mettons à l'épreuve après coup sur un terrain qui a été d'abord balisé par les cartographes du savoir. La théorie (in progress) du processus social d'Andrew Abbott nous sert de repère d'arrière-plan ${ }^{13}$. Il n'est pas lieu d'en résumer le contenu ni d'en épuiser les subtilités (éventuellement les difficultés) ${ }^{14}$. On peut dire, simplement, que l'approche d'Abbott nous est utile parce qu'elle accorde une grande importance à la question des frontières ou des limites (boundaries ${ }^{15}$ ); en effet, dans la dynamique de construction, de perpétuation/ reproduction et de disparition des entités (groupes, objets de représentation, etc.) peuplant le monde social, l'opération de traçage des frontières est antérieure à l'émergence des entités. En d'autres termes, les distinctions marquées par les acteurs créent les entités, en sorte que les frontières qui délimitent et ce faisant définissent celles-ci sont des conditions de possibilité ontologique. L'horographie prolonge ces idées sur le terrain de notre analyse. Nous montrons comment l'entité «Annales » résulte de pratiques de délimitation symbolique, épistémique, institutionnelle. L'identité de ce collectif, plastique sur le long terme, naît des différences marquées par rapport à d'autres entités, une certaine sociologie durkheimienne entre autres exemples.

Ainsi, nous pensons à partir d'un cas, sur la base d'une «sonde» historique, mais notre ambition est de contribuer à une problématisation plus générale des rapports interdisciplinaires, en fournissant des idées et des modes d'analyse de nature à éclairer d'autres réalités comparables. De plus, l'intention cognitive animant ce travail le positionne à l'intersection des problématiques de l'histoire conceptuelle, de l'épistémologie et de la sociologie des sciences sociales. Visant l'esquisse d'une théorie de l'horographie, il n'en demeure pas moins soumis aux mêmes mécanismes et processus. En sorte que le concept de frontière, qu'il s'agit d'élucider le plus précisément possible à travers ses usages, est l'objet et l'instrument d'une analyse réflexive par vocation autant que par nécessité.

Nous prenons en considération trois moments pendant lesquels la définition des rapports entre l'histoire et la sociologie fait problème. C'est dans ces circonstances que la cartographie des savoirs est modifiée, que l'autorité épistémique nécessaire au tracé des frontières est la plus disputée et mise à l'épreuve d'argumentations et de contreargumentations ${ }^{16}$. La démarcation prend des formes différentes suivant les contextes, c'est pourquoi il faut réinscrire chaque conjoncture horographique dans l'horizon de questionnement qui est le sien, sans jamais postuler a priori de continuités ${ }^{17}$. Parce

13. Аввотт, 1995 et 2001.

14. On trouvera une présentation des travaux d'Abbott dans FABIANI, 2003.

15. Nous n'entrerons pas dans les discussions concernant la traduction du mot «boundary» et des expressions formées sur cette base (boundary-work); automatiquement rapproché de la notion de frontière en français (et nous n'y coupons certes pas), boundary autorise des associations sémantiques différentes en version linguistique originale. Notre analyse des attendus et des impensés de l'imaginaire horographique fournira des éléments pour statuer sur les significations du mot « frontière ».

16. GIERYN, 2001.

17. On veut prévenir d'emblée les facilités de l'histoire à rebours, rétrospective et simplement téléologique, parce qu'elle induit une perception tronquée et vaguement présentéiste des réappropriations de la frontière entre sociologie et histoire. Cet avertissement de méthode est discuté par REVEL, 2007, p. 101. Il convient de noter que cet article de Revel, paru en 2007 dans la revue Mil neuf cent, avait déjà été édité en 1999 dans un ouvrage collectif allemand (voir REVEL, 1999). 
que l'histoire d'une revue ou d'une discipline est faite de ruptures de diverses natures (générationnelle, intellectuelle, politique, etc.) ${ }^{18}$ et les Annales l'illustrent sans aucun doute. Nous commençons par étudier la genèse d'une certaine politique de la connaissance historique, mise en place par les fondateurs des Annales. La distance critique prise avec la sociologie révèle une stratégie d'extension de l'espace de l'histoire façon Annales. Le deuxième temps de notre analyse correspond, dans les années 1950-1960, aux efforts de Fernand Braudel pour positionner les Annales dans un champ de sciences humaines et sociales en pleine mutation. Enfin, le troisième moment, la décennie 1980, concerne le renouvellement des axes épistémologiques dans un contexte de doute et de remise en question. Il n'est pas dans notre propos de faire l'examen des relations entre les Annales et la sociologie depuis leur fondation jusque dans les années 1990, mais plutôt d'envisager trois cas concrets et historiquement situés de mise à l'épreuve des frontières entre les savoirs. Et, afin de mieux saisir les enjeux, nous utiliserons comme point de repère et comme jalon les interprétations, les réponses (directes ou indirectes), les lectures contradictoires de l'article publié en 1903 par François Simiand dans la Revue de synthèse historique notamment contre l'école "méthodique » de Charles Seignobos ${ }^{19}$. Inlassablement convoqué, le texte est autant une source exemplaire pour les polémiques ultérieures qu'un embrayeur narratif.

HOROGÉNÈSES PRIMITIVES : LE « DÉBAT DE 1903 »
COMME RÉVÉLATEUR ET MOMENT D’ENCLENCHEMENT

Le réajustement de la frontière avec cette sociologie, voulue par le groupe des Annales au début des années 1930, est précédé, au début du $\mathrm{xx}^{\mathrm{e}}$ siècle, par un débat conflictuel entre les deux disciplines. À la fin du XIX ${ }^{\mathrm{e}}$ siècle, Émile Durkheim décrète l'urgence d'une réorganisation de l'espace cognitif des sciences sociales dans son ensemble. Dans la préface du premier volume de L'Année sociologique, paru en 1897, il plaide pour un rapprochement entre histoire et sociologie - mais pas à n'importe quelle condition. Durkheim estime en effet que pour « servir la cause de l'histoire », il importe « d'amener [celle-ci] à dépasser son point de vue ordinaire [...] à se préoccuper des questions générales que soulèvent les faits particuliers qu'ils observent ${ }^{20} \gg$. C'est en tendant vers l'abstraction, la modélisation et la constitution de lois, c'est-à-dire vers le corpus méthodologique et épistémologique de la sociologie prônée par l'auteur des Règles de la méthode sociologique que l'histoire serait utile aux sociologues. La volonté d'hégémonie affleure ici nettement, parce que Durkheim, après avoir demandé aux historiens d'adopter des conceptions qui leur sont étrangères, remarque que ce mouvement entraîne par nécessité une fusion des deux disciplines « en une discipline commune où des éléments de l'une et de l'autre se retrouveront combinés et unifiés ${ }^{21} »$. C'est sur le mode de l'injonction morale et de l'inéluctabilité que Durkheim légitime la division provisoire du travail intellectuel entre histoire et sociologie. Provisoire en

\footnotetext{
18. Hourmant, 2007.

19. SiMIAND, 1903.

20. DuRKHEIM, 1897, p. III.

21. DURKHEIM, 1897, p. III.
} 
ce sens que les frontières cognitives sont censées s'effacer à terme au profit d'une science sociale unifiée par la méthode. L'histoire se trouverait alors dépossédée de son identité propre, aliénée par un nomos épistémologique qui lui est étranger. La sociologie durkheimienne, encore fragile dans l'ordre institutionnel, s'affirme ainsi dans l'ordre des savoirs. La cohérence programmatique du groupe mené par Durkheim et sa volonté de saisir la société « dans son unité organique ${ }^{22}$ » poussent au conflit avec la discipline historique. Durkheim s'autorise en garant et annonciateur d'un nouvel ordre cognitif. C'est in fine le contrôle du discours sur la « société » (et la pertinence même du concept) qui établit la ligne de partage ${ }^{23}$.

Le débat de 1903 entre François Simiand et Charles Seignobos illustre un souci aigu de délimitation disciplinaire, entre une certaine sociologie en pleine ascension et les représentants de l'histoire dite " positiviste ${ }^{24}$. C'est, d'une certaine manière, la scène primitive des controverses qui nous intéressent. Proche des durkheimiens, Henri Berr ${ }^{25}$ saisit l'opportunité de publier, dans la Revue de synthèse historique qu'il a fondée en 1900, un article polémique de Simiand ${ }^{26}$ démontant l'ouvrage de Seignobos, $L a$ Méthode historique appliquée aux sciences sociales $(1901)^{27}$. En une quarantaine de pages, le philosophe critique l'histoire érudite qui s'attache au singulier et se refuse au général. Simiand plaide pour une « science des phénomènes sociaux ${ }^{28}$ ». Il prône l'abstraction, la comparaison systématique, révise à dessein le concept de causalité et réaffirme la prééminence ontologique du « social ». En insistant sur ces différents éléments, le philosophe-sociologue invite très clairement les historiens à rejoindre la discipline sociologique et s'emploie dans les années qui suivent à contrôler les commentaires dont son travail est l'objet.

C'est peu dire que Simiand est parvenu à produire un effet d'entraînement considérable et décisif. Point d'accrochage, l'article génère un flot de commentaires jusque vers 1908; comme on le verra, les débats à-venir s'y réfèrent de façon récurrente et il paraît aller de soi que les acteurs souhaitant intervenir sont supposés en connaître le fond. Ainsi, les commentaires sont autant de dérivations métatextuelles d'un textesource. On comprend dès lors pourquoi il constitue un révélateur des rapports de force. Les modes contraints par lesquels chacun s'approprie le discours de Simiand révèlent à tout moment l'état du champ intellectuel et les attendus des stratégies horographiques. Et plus largement encore, on peut mettre en relation le renvoi répété à l'article avec une dimension saillante de la dispute universitaire, à savoir, comme l'indique JeanLouis Fabiani, « l'importance du document ou du monument dans l'instauration d'une controverse ${ }^{29} »$.

22. DURKHEIM, 1888 , p. 48.

23. LeRoux, 1998, III part.

24. REBÉRIOUX, 1983. Jacques Revel note à juste titre que l'épithète « positiviste » qualifie négativement, stigmatise même la pratique historienne normalisée par Seignobos, alors même que celui-ci ne s'en prévalait pas; en réalité, le positivisme est plus à chercher chez les durkheimiens et leurs relais en histoire que chez leurs adversaires. Voir Revel, 2007, p. 114.

25. Fournier, 2007, p. 545-547. Voir aussi Prochasson, 1996.

26. Simiand, 1903.

27. Seignobos, 1901.

28. Cité par Aymard, 1988, p. 223.

29. FABIANI, 2007, p. 54. 
La question de la frontière entre histoire et sociologie au début du $\mathrm{xx}^{\mathrm{e}}$ siècle se noue autour des différences méthodologiques et des écarts épistémologiques. Simiand est alors un "jeune turc » militant de la sociologie qui se joue, avec une certaine arrogance, des asymétries institutionnelles. Comme l'indique Philippe Besnard, sa " position particulière [typique, autonome et orthodoxe] est à mettre en rapport avec ses engagements dans les polémiques épistémologiques et les luttes de territoires qui marquèrent l'émergence d'une nouvelle discipline ${ }^{30} »$. Attaquer l'approche de Seignobos ou de Hauser est un moindre mal intellectuel considérant les profits cognitifs escomptés - potentiellement convertibles au niveau institutionnel. Autrement dit, la critique épistémologique menée sous l'étendard de la "méthode» est, comme le signale Jacques Revel ${ }^{31}$, inséparable d'une forme de critique sociale: les durkheimiens énoncent les grandes lignes d'un réformisme intellectuel radical devant transformer les conditions d'exercice de la profession d'historien, en cours d'institutionnalisation sous la III ${ }^{\mathrm{e}}$ République. Simiand revendique une triple fonction d'arbitre, de régulateur-administrateur et d'instigateur. Certes, Seignobos occupe en Sorbonne une place prééminente dans le champ universitaire et, de manière générale, l'histoire est une discipline canonique représentée dans toutes les facultés. Il n'en va pas de même pour la sociologie qui veut se déprendre de sa filiation philosophique, et dont la visibilité universitaire est faible à l'aube du $\mathrm{XX}^{\mathrm{e}}$ siècle ${ }^{32}$. Quitte à provoquer un incident à la frontière et à prêter le flanc à l'accusation d'impérialisme, Simiand prend le risque de transporter en histoire la méthode positive de la sociologie. Un risque qui finit par payer vu l'isolement dans lequel Seignobos se trouve, parmi les historiens de surcroît ${ }^{33}$.

Une autre précision permet de rendre plus complexe la tension frontalière que les sociologues veulent imposer: la discipline historique n'est pas tout entière résumée aux thèses de Seignobos. Ainsi, comme l'a montré Laurent Mucchielli, il existe, dans les années 1890-1900, des « historiens professionnels, mais encore marginaux institutionnels ${ }^{34} \gg$ qui proposent de nouvelles orientations épistémologiques pour leur discipline. Charles Mortet défend une « histoire totale ${ }^{35}$ » qui chercherait des directions générales et des cadres explicatifs théoriques aux évolutions des sociétés. De même, le chartiste Paul Lacombe dans la préface de son ouvrage intitulé De l'histoire considérée comme une science, rompt avec l'érudition positiviste et souhaite que la collecte d'archives soit au service d'un questionnement portant sur les motifs et les mobiles psychologiques de l'action humaine ${ }^{36}$. Cette génération d'historiens marginaux, dans laquelle il faut inclure Pierre Caron, Henri Hauser, Paul Mantoux et surtout Henri Berr, n'a nécessairement pas entamée une mise en œuvre pratique et concrète des principes qu'elle défend - pour certains, le discours de la méthode se suffit à lui-même -; mais il faut remarquer que des linéaments de réponses au défi de Simiand existent à la périphérie de la discipline histoire. Cette dernière consiste dans un faisceau étendu de prises de

30. BESNARD, 2003, p. 311.

31. Revel, 2007, p. 116-120.

32. RebÉRIOUX, 1983, p. 221.

33. MucChielLi, 1998, p. 445-448.

34. MucChielLi, 1995, p. 69.

35. MucChielli, 1995, p. 70.

36. Mucchielli, 1995, p. 70. 
position qui, de façon plus ou moins expressément formulée, mettent en jeu des principes de vision des sciences sociales émergeantes ainsi que des modes d'identification disciplinaire ${ }^{37}$.

Cette brève description du positionnement de l'histoire et de la sociologie avant la création des Annales permet de saisir les écarts épistémologiques et institutionnels que les durkheimiens creusent et exploitent pour imposer un rapport de force sur la base d'un certain régime de scientificité et, par-là même, une frontière avec l'histoire, ou plus exactement avec une partie de la discipline. Il est d'emblée question d'une distribution des cartes entre les disciplines et, au-delà, d'identités disciplinaires. Dans le même temps, Victor Karady a montré que la stratégie de «légitimation de la sociologie » par un «travail collectif» et une grande cohérence épistémologique visait d'abord « à forcer l'octroi de positions ». Mais la Première Guerre mondiale, qui décime les rangs durkheimiens, "affaiblit le groupe de pression sociologique ». Dans le même temps, les normaliens se dirigent davantage vers des « disciplines plus immédiatement rentables pour leurs carrières ${ }^{38} »$. La faiblesse institutionnelle ne permet pas - malgré la position de force épistémologique, qui paraît relever en définitive du coup de force symbolique ou à tout le moins du bluff - de rendre efficiente la frontière tracée entre l'histoire et la sociologie, c'est-à-dire d'imposer aux historiens les termes mêmes du partage disciplinaire. Il n'empêche que le débat de 1903 ne cesse de réapparaître par la suite, sorte de retour du refoulé.

C'est en référence à cet arrière-plan intellectuel et institutionnel que Marc Bloch et Lucien Febvre fondent les Annales d'histoire économique et sociale. Laurent Mucchielli a très justement noté que les créateurs des Annales ont reconstruit leur propre généalogie scientifique en omettant les efforts de la génération d'historiens évoquée précédemment et qui avait tenté d'ouvrir l'histoire aux questionnements de la sociologie ${ }^{39}$. $\mathrm{Au}$ contraire, Bloch et Febvre ne cessent dans leurs écrits de connecter directement leur démarche aux travaux durkheimiens d'avant-guerre - des travaux dont l'esprit, d'après Johan Heilbron, s'est principalement répandu au-dehors de la sociologie dans les années 1920-1940 ${ }^{40}$. Bloch écrit ainsi dans les Annales en 1935: « [...] à la vieille Année [sociologique] les historiens de ma génération ont dû plus qu'ils ne sauraient dire $^{41}$. » Febvre reconnaît pour sa part la dette qu'il doit à la sociologie durkheimienne en ces termes:

«Lorsqu'on [avait] vingt ans [...] nous lisions L'Année sociologique, une des nouveautés qui retenaient le plus notre attention, n'était-ce point ce perpétuel effort de remaniement, de réadaptation des cadres de classement qui, de volumes en volumes, s'assouplissaient et se modifiaient [...]. Belle leçon de méthode qu'ils ne donnaient pas seulement à leurs fidèles déclarés: ils ont eu d'autres disciples $[\ldots]^{42}$. »

37. MuCChielLi, 1998, p. 425.

38. KARADY, 1976, p. 310 (toutes les citations précédentes sont tirées de la même page). Voir également KARADY, 1979.

39. MuCCHiElli, 1995, p. 69.

40. Heilbron, 1985 , p. 236.

41. Bloch, 1935 , p. 393.

42. FeBVRe, 1930, p. 583. 
Puisant aux sources durkheimiennes les éléments d'un renouvellement programmatique de l'histoire, Bloch et Febvre ont renoué avec le défi lancé par Simiand à l'endroit de leur discipline. Les deux historiens ont à plusieurs reprises exprimé leur dette envers le sociologue et tentent d'ailleurs de le convaincre de s'impliquer en sa qualité de spécialiste d'histoire économique dans les Annales dès $1929^{43}$. Febvre assure avoir été marqué par « l'influence parallèle ${ }^{44}$ » de celui qui, pour avoir « dit de dures vérités ${ }^{45}$ » aux historiens et formulé de la sorte « une critique d'une rare clairvoyance » «a pu sembler un noir ennemi de [la] discipline ${ }^{46} »$ historique. Bloch, quant à lui, défend dans son Apologie pour l'histoire, l'injonction de Simiand concernant la nécessaire direction (sociologique) à donner à toute enquête historique ${ }^{47}$. À partir du texte de 1903, les fondateurs des Annales dégagent les grandes lignes de leur projet éditorial: «La primauté de l'histoire-problème, la recherche de modèle, la convergence des sciences de l'homme et même l'invitation au travail collectif $[\ldots]^{48}$. » Jacques Revel remarque que l'article de Simiand apparaît " comme une sorte de matrice théorique ${ }^{49}$ " pour les Annales. Toutefois cette appropriation de certains éléments choisis de la sociologie durkheimienne ne doit pas occulter le souci d'extension épistémologique exprimé par les chefs de file des Annales, qui consiste à élargir toujours davantage le territoire des Annales et, dans le même mouvement, à contester le bien-fondé de la délimitation tracée à l'origine par Simiand. Bloch et Febvre s'emploient donc, dans une double stratégie inséparablement institutionnelle et épistémologique, à imposer leur lexique pour normaliser les écarts et les convergences entre sociologie et histoire. Leurs efforts pour produire une critique constante des institutions universitaires (agrégation, École des chartes) visent à imposer une marginalité qui, comme le souligne André Burguière «était plus tactique que réelle », les deux historiens étant « complètement [...] intégrés au système universitaire ${ }^{50} \gg$. Positionner une revue à la périphérie de l'institution permet d'opérer en surplomb de la communauté des historiens et, dans le même temps, de se rapprocher des autres sciences sociales: les Annales façonnent une zone cognitive de traverse à la confluence des champs disciplinaires. Cette tentative d'échapper à une indexation institutionnelle trop précise trouve son pendant épistémologique dans l'emploi du terme « social» dans le titre de la revue. Lors d'une conférence à l'École normale supérieure en 1941, Febvre explique à ce sujet « qu'un mot aussi vague que “social" semblait avoir été créé [...] pour servir d'enseigne à une Revue qui prétendait ne pas s'entourer de murailles mais faire rayonner largement, librement [...] sur tous

\footnotetext{
43. Voir « Une correspondance entre Lucien Febvre et François Simiand à 1'aube des “Annales” ", 1989.

44. FebVre, 1963, p. 311.

45. FebVre, 1930, p. 585.

46. Febvre, 1933, p. 163.

47. Sur ce point, voir NoIRIEL, 2005, p. 106.

48. ReVEL, 1979, p. 1364.

49. Revel, 1979, p. 1362. Revel revient sur cette «affirmation » dans l'article déjà cité (REVEL, 2007), comme nous le verrons dans le troisième moment de notre analyse.

50. Burguière, 1979, p. 1353. Les Annales bénéficient très directement de la position privilégiée de l'université de Strasbourg en France au lendemain du premier conflit mondial. Cet établissement, repris à l'Allemagne, est un enjeu symbolique majeur et fait l'objet d'une attention toute particulière de la part des sphères politiques et administratives. La Caisse nationale de la recherche soutient ainsi financièrement les publications de la faculté des lettres strasbourgeoise. Voir Dosse, 1987, p. 40.
} 
les jardins du voisinage, un esprit, son esprit $[\ldots]^{51} \gg$. Ce positionnement récuse implicitement la frontière qui séparait classiquement l'histoire "positiviste » et la sociologie durkheimienne. Il suppose également de ne pas insister sur les travaux de Paul Lacombe, Pierre Caron ou Henri Hauser.

C'est donc un nouveau rapport de force que les Annales tentent d'imposer à la sociologie. D'autant que cette dernière traverse, dans les années 1930, un « long purgatoire », selon l'expression de Jacques Revel et Roger Chartier. Son lien avec la philosophie l'empêche de conquérir une autonomie totale ${ }^{52}$. Il ne s'agit pas, pour les Annales, d'entrer en conflit permanent avec la sociologie mais au contraire de mener un lent travail de circonscription et de délimitation du territoire sociologique. L'ouverture à la sociologie s'exprime à travers le recrutement, au sein du comité de rédaction des Annales, de Maurice Halbwachs, leur collègue à l'université de Strasbourg. Le sociologue, naguère enrôlé par Simiand dans l'équipe de L'Année sociologique ${ }^{53}$, se trouve intellectuellement très proche de ses confrères historiens avec qui il partage jusqu'à un certain point le sens de l'interdisciplinarité ${ }^{54}$. Il a d'ailleurs fait ses gammes au moment même où la controverse de 1903 battait son plein. Comme l'indique Christian Topalov ${ }^{55}$, le travail du sociologue, en particulier sa thèse de droit sur Les Expropriations et le prix des terrains à Paris (1909), s'inscrit dans la lutte impérialiste partageant les durkheimiens et la «nouvelle école historique» de Seignobos et Hauser; l'enquête met précisément en œuvre le genre d' " empirisme rationaliste ${ }^{56}$ » que, selon Halbwachs, Simiand annonçait dès l'article de 1903. Seulement, le positionnement de Halbwachs au sein des Annales est singulier du fait même de sa discipline d'élection. L'éditeur de la revue - Armand Colin - est opposé à la venue d'un sociologue dans les instances dirigeantes des Annales et dans tous les cas, il n'est pas dans les objectifs des historiens de laisser la sociologie absorber le peu d'espace dont ils veulent disposer. Febvre indique ainsi qu'il n'est pas question de « donner dans la revue des articles de sociologie générale [...]», mais de faire de Halbwachs un « informateur » pour les historiens et, « dans une certaine mesure [un] critique ${ }^{57}$. De fait, on ne compte durant les dix premières années des Annales que six articles publiés par des sociologues ${ }^{58}$. En revanche Bloch et Febvre multiplient les comptes rendus d'ouvrages sociologiques; ils y détaillent ce qui sépare les Annales de la sociologie. En 1936, Bloch remarque à propos de la partie critique des Annales sociologiques de l'année précédente qu'elle est « trop abstraite [...] par endroits, pour notre goût d'historien, un peu trop portée aussi à passer sous silence ou à indiquer d'un simple mot, sans les discuter, des études relatives à notre passé européen, dont les enseignements ne sont pourtant pas négligeables ${ }^{59} »$. Le même Bloch défend, en 1934, le travail d'Eileen Power sur les études

51. FeBVRe, 1953, p. 20.

52. Chartier et Revel, 1979, p. 435.

53. BESNARD, 1979, p. 18.

54. Craig, 1979.

55. Topalov, 1997, p. 1068-1070.

56. Halbwachs qualifie ainsi le projet « doctrinaire » de Simiand dans un article apologétique paru un an après la mort de celui-ci (dans HaLBwACHS, 1936).

57. Cité par Leuillot, 1973, p. 320.

58. Craig, 1983, p. 273.

59. Bloch, 1936, p. 458. 
sociales parues en Angleterre la même année; il soutient avec elle que les sociologues négligent le matériau historique au profit d'analyses actuelles ou de recherches ethnographiques ${ }^{60}$. Cette critique est récurrente dans les premières années des Annales et Febvre la reprend lors de la $\mathrm{V}^{\mathrm{e}}$ Semaine internationale de synthèse, en 1933, pour illustrer les différences entre l'histoire qu'il défend et la sociologie ${ }^{61}$. L'absence de "profondeur » historique et le manque de perspective temporelle justifient - topoï rhétoriques confinant au stéréotype d'historien -, pour les fondateurs des Annales, une tension frontalière activée afin de maintenir à distance la sociologie durkheimienne. Et les historiens de reprocher de façon ironique aux sociologues de céder à la " généralité » décriée avec force par Durkheim dans Les Règles de la méthode sociologique. De l'autre côté de cette ligne Maginot intellectuelle, Halbwachs ne fait pas mystère du peu de crédit qu'il accorde à l'histoire malgré l'estime qu'il porte au travail de ses collègues « historiens sociologisants ${ }^{62}$ », une histoire qui est loin de pouvoir rivaliser scientifiquement selon lui avec la sociologie.

La vigilance horographique de Bloch et Febvre s'illustre également à travers la reformulation permanente des thématiques sociologiques en termes historiques (à l'exact inverse de ce qu'avait fait Simiand une trentaine d'années auparavant). La recension, par Bloch, du livre d'Halbwachs, Les Causes $d u$ suicide, indique bien ce renversement. L'historien salue l'usage du concept de « genre de vie » pour disséquer les types sociaux discernables sur la courbe du suicide. Il remarque que l'ouvrage s'inscrit dans une suite de productions récentes des durkheimiens qui récuse « toute décomposition excessive du complexe social entre les divers éléments que l'abstraction peut y distinguer ». Ces efforts répondent aux exigences des Annales et «aux besoins mêmes de tous ceux parmi les historiens qui, dans l'analyse du passé, répugnent à se contenter des définitions trop simplistes et tout extérieures que les schémas traditionnels offrent des différents groupes ${ }^{63} »$. Ainsi, les sociologues sont sommés de fournir des études conformes aux historiens des Annales, en modérant leur souci d'abstraction et en fournissant des définitions plus complexes de leurs objets d'étude. Or dans le même temps, cette capacité à saisir les innovations sociologiques utilisables par les historiens, permet aux Annales de se positionner comme pôle d'originalité et de renouvellement dans le champ historique et de viser son contrôle.

On mesure ici, entre les Annales et la sociologie, l'ampleur du redéploiement à la frontière opéré au profit de l'histoire. Le nouveau rapport de force repose à la fois sur un positionnement institutionnel subtil (l'histoire est une discipline très implantée dans le système universitaire et les Annales, tout en bénéficiant de ce point d'appui, s'affirment en marge de l'ordre académique), un emprunt direct aux registres épistémologiques utilisés par Simiand, une critique des visées trop généralistes et a-historiques des durkheimiens, une lecture « historienne » des travaux sociologiques et finalement une invitation à travailler pour l'histoire, au nom de la science sociale ${ }^{64}$.

60. BLOCH, 1934, p. 510.

61. Voir Craig, 1983, p. 275.

62. Selon l'expression de CRAIG, 1979, p. 283.

63. Bloch, 1931, p. 592.

64. Sur ce point, voir ReVEL, 2007, p. 123. 
À l'évidence, les Annales ne représentent pas - loin s'en faut avant guerre - toute la discipline historique ${ }^{65}$. De plus, la politique de délimitation n'est pas consensuelle au sein de la rédaction. Non seulement les modes de différenciation ne sont pas binaires (i.e. Annales vs sociologie), mais aussi les positions respectives de Bloch et Febvre ne sont pas nécessairement univoques et convergentes. Et, pour nuancer ce qui pourrait paraître relever d'une obsession sociocentrique, les déplacements transfrontaliers des deux historiens s'observent aussi à travers leur appropriation de la géographie (vidalienne) ${ }^{66}$; c'est là encore l'indice d'une stratégie d'ingestion interdisciplinaire qui n'a d'autre faim que consolider la discipline histoire. À l'intérieur même de l'institution des Annales, Febvre reproche à plusieurs reprises à Bloch ses incursions trop marquées en terres sociologiques. Par exemple, Bloch livre en 1941 le deuxième volume de sa Société féodale, Febvre en assure la recension dans les Annales. Il reproche à son collègue de ne dépeindre que des collectifs, des groupes d'individus. « Pourquoi pas de temps en temps, se détachant de la masse, un homme? Ou, si c'est vraiment trop demander, un geste d'homme tout au moins? », demande Febvre. Il poursuit en soulignant que le livre «marque, dans l'œuvre de Bloch, une sorte de retour au schématique. Nommons-le de son nom: vers le sociologique qui est une forme séduisante de l'abstrait ${ }^{67} »$. Le registre de justification des écarts repose sur des termes de sens commun intellectuel. Les couples d'opposition (abstrait/concret, passé/présent, etc.) informent une pratique de la démarcation d'autant plus efficace qu'elle essentialise l'insularité de pôles épistémologiques finalement irréductibles et prend acte d'une divergence vaguement esthétique en termes de «goût» disciplinaire. Et le travail de positionnement des frontières cognitives se double d'un souci de la distinction morale et symbolique, légitimant une hiérarchie de disciplines qui s'arrachent un objet, peu ou prou, commun.

Nous avons montré comment s'instaure une configuration horographique primitive à partir de la définition de Simiand et de ses réappropriations successives chez les historiens et les sociologues, spécialement par l'intermédiaire des Annales. La frontière se donne à voir comme l'enjeu matriciel de rapports de force socio-intellectuels fondés sur la perméabilité épistémologique des Annales et l'asymétrie des positions institutionnelles. Ces rapports de force ne sont pas, pour ce qui concerne la revue, monolithiques ou strictement homogènes. En effet, il existe à l'intérieur même des Annales des lectures différentes de la tension entre histoire et sociologie et des modes de tracés des frontières.

65. La Revue historique constitue (à la même époque que les Annales) le point d'ancrage d'une histoire-bataille traditionnelle qui reste dominante. La Revue historique est une publication de référence au moment où les Annales émergent; il faut aussi compter avec la Revue d'histoire moderne et contemporaine et la Revue de synthèse. Le paysage éditorial ne se réduit donc pas aux positions des Annales (Dosse, 1987, p. 45-53). C'est d'autant plus vrai que les inflexions interdisciplinaires des historiens des Annales peuvent, si elles sont mises en avant, constituer une entrave sérieuse à la poursuite de la carrière. Ainsi, lorsque Lucien Febvre se présente en 1926 à la Sorbonne sur la chaire de Charles Seignobos, il est battu par Raymond Guyot dont le profil est plus classique (NoIRIEL, 2005, p. 330-331).

66. Voir Friedman, 1996.

67. FeBVRE, 1941, p. 128. 
BIFURCATIONS. FRANCHIR ET DISSOUDRE LA FRONTIÈRE

Le deuxième moment que nous examinerons entre l'école des Annales et la sociologie intervient dans les années 1950 et 1960. Les Annales sont alors indirectement puis directement contrôlées par Fernand Braudel qui y impose ses thèmes (la géohistoire, l'analyse des cycles) et ses méthodes (la division tripartite du temps). Comme l'ont montré François Dosse et André Burguière, au lendemain de la Seconde Guerre mondiale, les Annales sont devenues hégémoniques dans le champ historique; recrutements et publications recoupent très largement les choix et les problématiques de la revue ${ }^{68}$. Le « monde braudélien ${ }^{69} "$, selon l'expression de Jack Hexter, opère une jonction entre les entreprises institutionnelles ${ }^{70}$ de l'auteur de La Méditerranée (notamment la création de la Maison des sciences de l'Homme, la structuration de l'École des hautes études en sciences sociales, la présidence du jury d'agrégation d'histoire) et le redéploiement programmatique des Annales autour des grandes enquêtes quantitatives de l'histoire économique. Le paysage institutionnel des sciences humaines et sociales s'est alors totalement transformé. En effet, comme le remarque Jacques Revel, ce n'est que dans les années 1950 et surtout dans les années 1960 que ces disciplines acquièrent une reconnaissance leur permettant de s'affirmer dans le système universitaire (postes, filières, diplômes) ${ }^{71}$.

François Simiand est devenu une référence intégrée - par l'entremise d'Ernest Labrousse en particulier -, un emblème qui n'est pas discuté. L'article de 1903 est publié dans les Annales en 1960, alors que Fernand Braudel en assure la direction. Le texte est reproduit in extenso afin que les jeunes historiens puissent « mesurer le chemin parcouru en un demi-siècle » et « comprendre ce dialogue de l'Histoire et des Sciences sociales, qui reste le but et la raison d'être de la Revue ${ }^{72} »$. L'article qui avait si profondément remis en question l'histoire " positiviste » fait ainsi désormais partie du patrimoine des Annales braudéliennes qui, à travers la réédition du texte, marque, comme nous allons le voir, la volonté d'abolir la frontière avec la sociologie au profit d'une forme de pandisciplinarité conséquente et conquérante ${ }^{73}$.

Le structuralisme participe lui aussi du point de vue épistémologique à une remise en cause des rapports de force entre les sciences humaines et l'histoire. Claude LéviStrauss, en particulier, recherche les expressions inconscientes des sociétés qui se manifestent dans des invariants et des formes immuables ${ }^{74}$. Cet examen d'une nature humaine ancrée dans l'universel est une démarche résolument a-historique, sinon antihistorique. Braudel perçoit un rejet massif de l'histoire soudant les sciences humaines

68. Dosse, 1987, p. 118-127.

69. HeXTER, 1972.

70. La maîtrise des financements, lors du développement de la $\mathrm{VI}^{\mathrm{e}}$ section de l'École pratiques des hautes études puis de la création de l'École des hautes études en sciences sociales, constitue l'un des points forts de l'entreprise institutionnelle braudélienne. Les Annales sont adossées à une structure universitaire renforcée et bien dotée (Dosse, 1987, p. 118-127). Voir aussi DAIX, 1995, p. 285-323.

71. Revel, 1995, p. 75.

72. Dans Simiand, 1903, ici 1960, p. 83, note de la rédaction.

73. HaLl, 1999, p. 173.

74. LÉvi-Strauss, 1949. 
et sociales entre elles. C'est pourquoi il produit une série de discours à l'intention notamment de l'anthropologie et de la sociologie. Concernant cette dernière, c'est avec Georges Gurvitch que Braudel entame une discussion critique. En 1953, Braudel fait paraître un article dans les Annales ${ }^{75}$ qui remet en cause les cinq architectures essentielles au moyen desquelles Gurvitch décrit l'édifice social dans sa globalité grandiose ( « les paliers en profondeur, les sociabilités, les groupes sociaux, les sociétés globales, les temps $\left.{ }^{76} »\right)$. Cette multiplicité et cette fragmentation font craindre à l'historien la dissolution du paradigme unitaire qu'il défend: la société doit être examinée comme un tout ${ }^{77}$. Il retrouve en ce sens l'intention originelle d'un Durkheim (i.e. l'idée d' « unité organique ») court-circuitée par ses propres continuateurs.

Une deuxième confrontation en 1957-1958, plus franche encore, permet de mieux saisir les ambitions, mais aussi les contradictions de Braudel à l'endroit de la sociologie. Gurvitch publie en 1957 un texte dans les Annales intitulé « Continuité et discontinuité en histoire et en sociologie ${ }^{78} »$. Il prévient d'emblée les malentendus en soulignant qu' " histoire et sociologie ont le même domaine, celui des phénomènes sociaux totaux et des hommes totaux ${ }^{79}$ ». Néanmoins, il ne manque pas de souligner, dans la foulée, les écarts méthodologiques entre les disciplines: «[...] la méthode de la sociologie est typologique, celle de l'histoire est individualisante ${ }^{80}$. $\gg$ Le sociologue considère que le temps historique est à la fois «accompli et reconstruit ${ }^{81}$ »; il met en valeur les singularités davantage que les effets de structures; enfin, il intensifie la spécificité du lien causal. L'histoire est donc, pour lui, continuiste ${ }^{82}$ et s'oppose, sur ce point précis, à la sociologie qui privilégie les discontinuités et les ruptures. En conclusion, Gurvitch soutient que l'histoire et la sociologie sont « en rapport dialectique très net » et que, «selon leurs phases, elles peuvent se trouver en rapports de complémentarité, d'implication mutuelle, d'ambiguïté, de polarisation, de réciprocité de perspective ${ }^{83} \gg$. L'article du sociologue signale en termes clairs les divergences épistémologiques qui séparent les deux disciplines.

Un court texte de Braudel prévient les tentatives du sociologue de tracer une frontière entre histoire et sociologie depuis sa discipline. Il s'agit d'un court-circuit au sens strict puisqu'il prend la forme d'un incipit en italique placé entre le titre et le corps de l'article de Gurvitch et qu'il est signé des initiales F. B., comme s'il s'agissait d'une relecture " amicale » traquant les insuffisances d'un texte encore à l'état d'ébauche. Braudel souligne que Gurvitch tente « une fois de plus de marquer l'indécise frontière entre les travaux, les pensées et les tendances de ces deux amis en querelle constante: le sociologue et l'historien ${ }^{84} »$. Refusant le tracé de cette frontière professionnelle,

\footnotetext{
75. Braudel, 1953.

76. BRAUDEL, 1969 , p. 78.

77. BRAUDEL, 1969, p. 78.

78. Gurvitch, 1957.

79. GuRVITCH, 1957, p. 74.

80. GuRVITCH, 1957, p. 74.

81. GuRVITCH, 1957, p. 81.

82. GuRVITCH, 1957, p. 81.

83. GuRVITCH, 1957, p. 83.

84. Dans Gurvitch, 1957, p. 73 (il s'agit de l'incipit de Braudel).
} 
Braudel assure que là où « Georges Gurvitch cherche des différences, des désaccords, nous avons toujours vu des analogies, des rencontres et un travail commun. Histoire et sociologie ne sont pas pour nous, l'envers et le revers d'une étoffe, mais bien cette étoffe dans toute son épaisseur $[\ldots]^{85} \gg$. Cette incise contradictoire n'est en fait que la prémisse d'un célèbre article de Braudel («Histoire et science sociale: la longue durée ${ }^{86} »$, publié en 1958), qui fait le point sur le positionnement de l'histoire à l'égard des autres sciences humaines et sociales, mais qui est également une réponse argumentée à l'article de Gurvitch édité l'année précédente. Braudel prend d'abord acte de l'indépassable géopolitique des savoirs qui traverse l'ensemble des sciences humaines et sociales: "Les voilà, à l'envi, engagées dans des chicanes sur les frontières qui les séparent ou ne les séparent pas $[\ldots]^{87}$. » Il remarque qu'en cherchant à saisir « le social en son entier», chaque science sociale « empiète sur ses voisines en croyant demeurer chez elle ${ }^{88} »$. Les notions de frontière et de territoire disciplinaire constituent la topique de l'historien en même temps que l'arrière plan de son argumentaire. Son premier soin est de dessiner (ou redessiner) les contours de la discipline histoire. Il la décrit comme "peut-être la moins structurée des sciences de l'homme ", capable d'accepter « toutes les leçons de son multiple voisinage » et de « répercuter ${ }^{89}$. Plastique et ouverte, l'histoire déborde de ses frontières et se nourrit de concepts étrangers et de méthodes exogènes - dans l'esprit, à tout le moins, elle n'est pas éloignée de l'histoire de Bloch. Dans la quatrième et dernière partie de son article, Braudel s'attache plus précisément à sonder les écarts et les convergences entre l'histoire et la sociologie. Il critique la manière dont les sociologues envisagent le temps: «[...] leur temps n'est pas le nôtre, il est beaucoup moins impérieux, moins concret aussi, jamais au cœur de leurs problèmes et de leurs réflexions ${ }^{90}$. » Le temps des sociologues est décrit comme « complaisant ${ }^{91}$ » et sécable à l'envi. Braudel érige la temporalité et la question de la durée comme principe épistémologique premier de l'histoire. Cet axiome de portée ontologique de la recherche historique est absent selon lui de la sociologie; il n'apparaît pas dans le corpus méthodologique des sociologues. Le temps, « cette contrainte à laquelle l'historien n'échappe pas, les sociologues, eux, y échappent presque toujours : ils s'évadent ou dans l'instant présent, toujours actuel, comme suspendu au-dessus du temps, ou dans les phénomènes de répétition qui ne sont d'aucun âge ${ }^{92} »$, écrit Braudel. C'est ici que l'article apparaît comme une réponse indirecte à Gurvitch qui reprochait aux historiens leur continuisme. On saisit dès lors le jeu de frontières entrepris par Braudel. Il ne revient pas sur la critique de Gurvitch, mais puise au cœur même de la démarche historique une donnée heuristique (la réflexion sur la durée) pour montrer qu'elle manque aux sociologues et formuler dans ses propres termes (d'historien)

85. Dans Gurvitch, 1957, p. 73 (il s'agit de l'incipit de Braudel).

86. BRAUdEL, 1958. Nous citerons par la suite le texte inséré dans l'édition des Écrits sur l'histoire (BRAUDEL, 1969).

87. BRAUDEL, 1969, p. 41.

88. BRAUDEL, 1969, p. 42.

89. BRAUdel, 1969, p. 42.

90. BRAudel, 1969, p. 75.

91. Braudel, 1969, p. 77.

92. Braudel, 1969, p. 79. 
l'écart entre les deux disciplines. Il peut sembler paradoxal de signifier clairement les différences entre champs de recherche et, dans le même temps, d'appeler à la convergence voire à la coopération. Or, la contradiction n'est qu'apparente. Si la durée est le centre de gravité épistémologique de l'histoire (et donc ce qui la caractérise), elle peut aussi être projetée dans toutes les sciences humaines et sociales. À elles (et notamment la sociologie) d'imiter l'histoire (capable d'intégrer toutes les méthodes extérieures) et d'incorporer la réflexion sur le temps à leur démarche. La dispute sur le temps a pour effet de dramatiser les réarrangements disciplinaires ${ }^{93}$.

Fernand Braudel formule cette invitation à vertébrer la sociologie par la durée, dans le chapitre IV de l'introduction du Traité de sociologie, publié entre 1958 et 1960, sous la direction de Georges Gurvitch. L'historien écrit que «le temps, la durée, l'histoire s'imposent en fait, ou devraient s'imposer à toutes les sciences de $l^{\prime}$ 'homme ${ }^{94} »$. Braudel poursuit son raisonnement en constatant qu'avec l'intégration de la problématique temporelle «l'histoire et la sociologie se rejoignent, s'identifient, se confondent ${ }^{95} »$. Les deux disciplines n'en feront alors qu'une. On mesure le travail aux frontières accompli ici par Braudel (et qui rappelle, en termes inverses, celui de Durkheim à la fin du XIX ${ }^{\mathrm{e}}$ siècle): dans le paysage disciplinaire mouvant des années 1950-1960, où l'histoire est rejetée par certaines sciences humaines et sociales, l'auteur de La Méditerranée s'efforce de maintenir le leadership de sa discipline, désormais très largement configurée aux formes de la revue qu'il dirige, en définissant et en occupant des structures institutionnelles puissantes (École des hautes études en sciences sociales, Maison des sciences de l'Homme). Dans le même temps les Annales (son point d'appui principal), lui permettent d'avancer une argumentation unificatrice vers les autres disciplines des sciences humaines et sociales. Braudel s'emploie, avec la sociologie, à réduire les écarts en même temps qu'il formule son irréductibilité à l'histoire, par l'absence de réflexion sur le temps. Il tente donc de souligner une différence épistémologique nette entre la sociologie et l'histoire (la durée), tout en enjoignant la sociologie à s'en emparer et par-là à se fondre dans l'histoire-intégratrice. Braudel pointe donc une frontière (définie depuis le corpus épistémologique de l'histoire telle que les Annales l'ont forgé après guerre) et propose aux sociologues de la dissoudre en acceptant la réflexion sur le temps. Il évite de s'enliser dans un dialogue de sourds avec Gurvitch $^{96}$. Le jeu dans le tracé des frontières révèle les craintes du chef de file des Annales de voir la discipline que sa revue incarne désormais presque totalement être débordée par les autres sciences humaines et sociales, en même temps qu'une réactivation de l'histoire comme discipline canonique et hégémonique.

93. Sur ce point, voir MaILlaRd, 2005.

94. BRAUDEL, 1969, p.105.

95. BRAUDEL, 1969, p. 105.

96. Les jeux sont faits bien avant la passe d'arme théorico-rhétorique de 1957-1958. En 1955, Braudel fait un éloge plus qu'ironique de la contribution de Gurvitch à l'histoire lors d'une conférence donnée au Collège philosophique. Il considère ainsi que « Gurvitch a rendu, beaucoup plus qu'il ne le pense, des services éminents à la cause des historiens dans la mesure où il a fait, en arrière de nous, un magnifique barrage d'artillerie, d'explosifs. Non seulement il a mis à mal toute la sociologie du $\mathrm{XIX}^{\mathrm{e}}$ siècle, mais comme l'explosion n'a pas été très bien dirigée (peut-être, $\mathrm{j}$ 'en ai peur, intentionnellement), toute la pensée du début du $\mathrm{Xx}^{\mathrm{e}}$ siècle a été dynamitée en même temps » (texte de la conférence «L'impérialisme dans l'histoire » repris dans BraUdeL, 1997, p. 135-136). 
Gurvitch demeure vigilant et engage le débat. Il ne cesse de pointer la singularité de la sociologie parmi les sciences de l'Homme. Et même s'il se défend d'une quelconque «prétention impérialiste » à la manière de l'école durkheimienne et s'emploie à l'inverse à mettre en valeur la pluralité des méthodes par-delà les disciplines ${ }^{97}$, il n'en demeure pas moins sous-entendu dans son argumentaire qu'elle occupe une place prédominante en raison de sa « complexité » intrinsèque - tant sur le plan de sa méthode que de ses objets ${ }^{98}$. Ses réflexions, toujours systématisantes, visent l'ordre et le classement. De la même façon qu'il identifie des «étagements » de la société, Gurvitch, en infatigable taxonomiste de la science sociale, assigne à chaque discipline la place qui doit lui revenir et s'applique à justifier ses résolutions horographiques; par exemple, dans le premier chapitre du monumental Traité de sociologie intitulé « objet et méthode de la sociologie » $(1958)^{99}$. L'usage de la dialectique s'avère ici très stratégique. Gurvitch s'en sert comme d'une arme rhétorique dans l'échange d'arguments. Dans Dialectique et sociologie (1962), il aiguise une fois de plus les « procédés dialectiques opératoires » sur le problème des rapports entre histoire et sociologie. L'hyperdialecticien est au-dessus des procédés dialectiques qu'il identifie au préalable ${ }^{100}$. Il prétend dépasser le «dogmatisme » de ces schèmes de pensée exclusifs et pense ainsi se démarquer des chercheurs qui, à l'exemple de Braudel, persévèrent dans une dialectisation limitée et partielle. D'après ses analyses, l'historien se satisfait en effet de la « réciprocité de perspectives » de l'histoire et de la sociologie; or, son souci (manifeste) de convergence disciplinaire trahit selon Gurvitch une adhésion utopique à un " "idéal" non atteint... et difficile à atteindre ${ }^{101}$ ». Et le sociologue d'en appeler à l'instauration d'un « duumvirat fraternel » de l'histoire et de la sociologie, qui annoncerait l' « intégration » de l'ensemble de la «Science de l'Homme » ${ }^{102}$. L'intention généreuse, qui balaie les suspicions d'impérialisme sur fond d'hyper-réalisme dialectique, ne parvient pas à masquer cependant la différenciation opérée dans la pratique. Qu'il s'en défende, le sociologue n'en utilise pas moins dans ses analyses le procédé de dialectisation par «polarisation dialectique » (pour reprendre ses propres catégories). Il n'opère pas autrement que les horographes qui n'ont de cesse d'intensifier l'« inflation artificielle des antinomies » et le « fétichisme » qui lui est pendant ${ }^{103}$. Il prête des qualités immuables aux disciplines et en éternise les orientations théoriques. Parce que la dialectisation, fût-elle polarisante, demeure un mode d'essentialisation. Sociologie et histoire se trouvent caractérisées dans leur essence et préservées dans leur différence; n'en déplaise à Braudel, elles ne sont pas taillées dans la même étoffe. La volonté d'hégémonie de Gurvitch s'affirme à travers un type subtil de travail de démarcation épistémologique effectué dans les nomenclatures du savoir. Il s'autorise pour ce faire

97. GuRVITCH, 1958, p. 10.

98. Gurvitch, 1958, p. 3.

99. GuRVITCH, 1958.

100. GuRvitch, 1962, p. 224-232. Il en dénombre quatre: l'« éclairage par complémentarité réciproque », la « complémentarité dialectique », la « polarisation dialectique » et enfin la « réciprocité de perspectives ».

101. Gurvitch, 1962, p. 232.

102. GuRVITCH, 1962, p. 232.

103. GuRVitch, 1962, p. 207-208. 
d'un méta-procédé se voulant objectif - à la limite, transcendantal - et ainsi d'autant plus imparable contre les essais d'épistémologie appliquée d'un Braudel. Par-dessus tout, l'auteur ne s'empêche pas de parler (seul) au nom de la discipline sociologique (au singulier): il l'incarne, l'oriente, la régit. Elle est la «Science de l'Homme » et l'histoire n'en est qu'une branche.

Il convient - pour terminer sur ce point - de relativiser les effets performatifs de ce jeu de frontières élaboré depuis les Annales. Maurice Aymard constate en effet qu'après les années 1960, les historiens «paraissent accepter [la] division des champs d'étude et de compétences avec les sociologues ${ }^{104}{ }$ ) et se tournent alors vers des périodes plus anciennes. L'appel de Braudel n'est suivi ni par les sociologues ni par les historiens. Là n'est pas son ambition. L'incandescence des frontières est d'abord discursive et, même si les conséquences de ces réflexions programmatiques sur les écarts et les convergences ne sont pas nulles dans la structuration et l'organisation des savoirs, il ne faut pas surestimer les conséquences concrètes et pratiques (sur les institutions et les contenus cognitifs) d'une rhétorique de la délimitation qui matérialise davantage des rapports de force en cours - un différend interindividuel, dirions-nous - que des reconfigurations étendues réellement à l'œuvre. Les tactiques d'intimidation n'ont d'autre fonction pour les horographes que de mesurer leur force et celle de l'adversaire.

La présence spectrale de l'argument de Simiand est marquante. C'est dans les moments de crise épistémologique (peu importe ici qu'elle soit réelle, perçue ou anticipée) que le refoulé durkheimien réapparaît. Pierre Bourdieu affirme - à dessein - à ce sujet que les «successeurs [des fondateurs des Annales] n'ont pas cessé de reproduire ce refoulement, avec une belle constance ${ }^{105} »$. Patrimoine paradoxal de l'histoire façon Annales, l'article n'en sort pas moins métamorphosé en tant qu'il sert de nouvelles attentes cognitives. Mais il en a perdu aussi au passage son intensité dramatique et sa charge polémique. Car si les historiens savent qu'il est a priori disponible et lisible, ils ne le prennent plus tout à fait au mot ni pour argent comptant. Le texte s'efface peu à peu dans les notes de bas de page, à peine cité tant il est " célèbre » et «bien connu » (entre autres qualificatifs sur-utilisés). Non pas qu'il ait perdu totalement sa magnitude intellectuelle: il est bien plutôt un résidu d'une science désormais froide. Aussi le retour de Simiand ne s'opère qu'à la faveur d'une forme de distanciation affective et d'un remaniement discursif: l'article est une pièce muséologique, un " document ", un support mémoriel - et son auteur un personnage conceptuel. Il n'en demeure pas moins que l'usage qu'en fait Braudel n'est pas exempt non plus d'arrière-pensées stratégiques, comme l'a récemment deviné Revel ${ }^{106}$ : en le réinjectant tel quel dans les Annales, l'historien réaffirme l'inanité des tentatives d'unification monodisciplinaire d'où qu'elles viennent (de la sociologie de Gurvitch ou du projet structuraliste) au profit d'une interdisciplinarité confraternelle - et fait passer ainsi son propre projet pour ce qu'il n'est pas.

104. AYMARD, 1988, p. 228.

105. Bourdieu, 1995, p. 109.

106. Revel, 2007, p. 124-126. 
RÉFLEXIVITÉ HOROGRAPHIQUE: SE RÉAPPROPRIER LA FRONTIÈRE

Les années 1980 sont marquées, pour les Annales, par un double mouvement de remise en cause et de renouvellement. Les critiques - internes et externes au champ de l'histoire - ainsi que l'essoufflement du paradigme quantitatif conduisent la revue à plusieurs exercices d'auto-analyse et de réévaluation des axes de recherche. Dans ce travail introspectif et prospectif, le texte de Simiand est mobilisé à de nombreuses reprises; il permet de renouveler, d'actualiser et finalement de reformuler la question de la frontière avec la sociologie. Deux épisodes nous permettrons de mieux saisir ce travail de réappropriation du cadrage disciplinaire: le numéro marquant les 50 ans de la revue en 1979, et celui envisageant en 1989, sous le titre « Tentons l'expérience », la place de la revue dans le paysage historiographique remodelé.

Le texte présentant, en 1979, le demi-siècle des Annales indique que « l'histoire et le métier d'historiens sont confrontés à des problèmes nouveaux ${ }^{107}$ ». Outre les questions relatives au recrutement et à l'enseignement de la discipline, un bilan des rapports avec les autres disciplines est esquissé: les liens sont désormais plus lâches avec la géographie et l'économie; ils se sont resserrés avec l'anthropologie. La sociologie n'est tout simplement pas évoquée. Jacques Revel, dans l'article qu'il consacre quelques pages plus loin aux « paradigmes des Annales », analyse l'histoire de la controverse de 1903 et son empreinte sur les fondateurs de la revue. Il déclare que l'article constitue « une critique serrée » de l'école méthodique, une influence indéniable pour Lucien Febvre, Marc Bloch, Ernest Labrousse, Fernand Braudel et Jean Bouvier, un texte « recevable » au moins « formellement » sur la définition des rapports entre l'histoire et les autres sciences sociales, ainsi qu'une matrice dans laquelle les historiens des Annales ont puisé pour définir et proposer leurs orientations (i.e. histoire problème, modèles explicatifs, travail collectif) ${ }^{108}$. La reconnaissance de cette dette à l'égard de Simiand est toutefois contrebalancée par une lecture historique de l'article du sociologue qui rappelle les enjeux conjoncturels liés à sa publication. Revel souligne que l'article n'était pas isolé et qu'à l'étranger les rapports entre l'histoire et la sociologie sont également interrogés à l'époque ${ }^{109}$. L'historien remarque que le texte de Simiand est « provocateur », « injuste » pour les historiens qui, dès la fin du XIX ${ }^{\mathrm{e}}$ siècle, ont proposé des pistes épistémologiques se démarquant de l'histoire méthodique de Seignobos ${ }^{110}$. Il voit donc dans la production de Simiand un "manifeste », un " article de circonstance », une œuvre de combat: en 1903, la sociologie est une discipline marginale dans le champ académique et les durkheimiens sont dans une phase de « conquête ${ }^{111}$. Cette mise à distance critique de l'article de Simiand, par le biais d'une lecture historique éclairant les enjeux institutionnels et cognitifs de l'époque, permet à Revel de réévaluer l'horographie effectuée dans les années 1930 par les Annales pour incorporer

107. « Les Annales, 1929-1979», 1979, p. 1344.

108. REVEL, 1979.

109. ReVEL, 1979 , p. 1376

110. ReVel, 1979, p. 1363.

111. ReVEL, 1979, p. 1363-1364. 
le durkheimisme au corpus épistémologique de ces nouvelles pratiques historiques. L'auteur souligne que Febvre - en particulier - ne se plie pas, loin s'en faut, à toutes les exigences formulées par Simiand à l'endroit de l'histoire: ainsi Febvre « revendique la spécificité de son approche et la nécessité d'une dimension historique dans toute réflexion sur les objets sociaux $[\ldots]^{112} \gg$. Le constat est sans appel: Revel constate une déformation progressive du projet de Simiand pour l'histoire - et pas n'importe laquelle - notamment dans l'unification souhaitée des « sciences sociales » autour du raisonnement sociologique ${ }^{113}$.

On mesure combien ce retour sur l'histoire du tracé de la frontière entre la sociologie et les Annales permet, en 1979, d'actualiser un clivage, de signifier, par le questionnement de l'héritage Febvre-Bloch, la permanence d'une irréductible différence entre la démarche sociologique et la démarche historique. Les succès passés des Annales avaient masqué les démarcations disciplinaires que la remise en cause de l'hégémonie historienne à l'aube des années 1980 permet de réactiver. Rappeler la persistance d'une frontière avec la sociologie, faire ressortir le clivage épistémologique dans l'histoire (historienne) des Annales, en rappeler l'irréductible présence, permet, dans une période où le jeu des coordonnées de la cartographie disciplinaire est renouvelé, de poser en principe la spécificité de l'histoire et surtout celle de la revue. Il n'est plus question d'abolir les écarts épistémologiques, comme lorsque Fernand Braudel invitait les sociologues à s'emparer de la durée pour rejoindre les historiens selon un horizon pandisciplinaire, mais bien au contraire de les revendiquer en montrant leur permanence. De sorte que la légitimité des Annales se jouerait dorénavant sur le territoire de l'histoire. On comprend alors pourquoi la définition des contours et des fins de la discipline est donc une impérieuse nécessité.

La fin des années 1980 constitue pour les Annales une période de recomposition des problématiques de la revue et de repositionnement épistémologique ${ }^{114}$. La rédaction propose en 1988 d'ouvrir une réflexion sur le « reclassement des disciplines » pour aborder un « tournant critique ${ }^{115} »$. L'« ambition fédératrice» de l'histoire n'est plus d'actualité, il convient désormais d'interroger la «crise générale des sciences sociales ${ }^{116} \gg$. Un an plus tard, à la fin de l'année 1989, un numéro complet des Annales rend ainsi compte des réflexions en cours tout en fournissant un panorama surplombant des champs d'intervention possibles pour la revue à l'avenir. L'éditorial met en avant l'heuristicité des transports disciplinaires et des échanges conceptuels. Il ressort de cette focale la priorité de la matrice disciplinaire comme point de référence à partir de laquelle s'articulent les propositions. Certes, les rédacteurs ont conscience du fait que le décloisonnement des savoirs est une antienne des Annales depuis sa fondation. N'inversant pas ce programme, l'objectif consiste encore à « dessiner le champ d'une confrontation fructueuse des investigations en cours [et de] cristalliser les nouveaux questionnements $[\ldots]^{117} »$. La revue souhaite réévaluer les moyens et les fins d'une

112. ReVEL, 1979, p. 1365.

113. ReVEL, 1979, p. 1368.

114. Delacroix, Dosse et Garcia, 2007, p. 513.

115. « Histoire et sciences sociales. Un tournant critique?», 1988, p. 291.

116. « Histoire et sciences sociales. Un tournant critique?», 1988, p. 292.

117. « Tentons l'expérience », 1989, p. 1317. 
interdisciplinarité jugée jusqu'ici sans appuis méthodologiques stables ${ }^{118}$. Les Annales font le constat qu'en 1989 « aucune discipline ne peut prétendre à une hégémonie intellectuelle ou institutionnelle sur les sciences sociales ${ }^{119} »$. C'est pourquoi il est inutile de chercher dans ces conditions à élaborer un paradigme unitaire capable de rassembler ces sciences. La propension à l'unification et à la fondation d'une science " globale »a fait long feu. L'acceptation d'une indépassable fragmentation épistémique des sciences de l'homme est signe de maturité: sorties de l'enfance et des prétentions monopolistiques, les historiens se font à la conjoncture interdisciplinaire prévalant alors. En effet, la revue envisage plutôt de dessiner une interdisciplinarité souple (Bernard Lepetit parlera d'une « interdisciplinarité restreinte $\left.{ }^{120} »\right)$ dans laquelle chaque discipline est fermement adossée à ses pratiques, ses outils heuristiques et ses thématiques motrices. Les Annales refusent de penser « la relation entre disciplines en termes d'homologie ou de convergence ${ }^{121} »$; elles plaident au contraire pour une affirmation des spécificités qui permettent ensuite des confrontations de pratiques et de problématiques. Le dialogue entre les disciplines (et l'histoire n'est ici plus mise en avant dans la configuration du dialogue, elle apparaît comme une discipline parmi les autres, au même titre que les autres) doit par conséquent être envisagé comme une multiplication des regards, une « mise à distance critique de chacun des modes de représentation du réel ${ }^{122}$ » sans sousentendu hégémonique. Ce point de vue œcuménique est aussi contraint par l'espace des possibles en matière d'horographie disciplinaire. Les configurations disciplinaires (cognitives et institutionnelles) des années 1990 interdisent en effet aux Annales toute tentative de domination du champ des sciences humaines et sociales comme cela avait été le cas auparavant. L'affirmation d'un nécessaire retour aux identités tranchées des disciplines pour permettre ensuite des échanges fructueux marque, pour les Annales, un état de stabilité et de reconnaissance mutuelle des frontières et des discontinuités entre ces disciplines.

Il n'empêche que la sociologie a voix au chapitre dans le numéro spécial des Annales. Un texte de Gérard Noiriel propose une clarification des termes des discussions qu'historiens et sociologues peuvent (et devraient) selon lui entamer ${ }^{123}$. Il revient d'abord sur les « impasses d'une vieille querelle ${ }^{124}$ » entre sociologie et histoire. Il retrace les origines du débat entre sociologues durkheimiens et historiens positivistes en soulignant le poids originel des « lignes de fractures ${ }^{125}$ » pointées au début du $\mathrm{Xx}^{\mathrm{e}}$ siècle. Le texte de Simiand est, une nouvelle fois, mis à contribution pour éclairer les enjeux du débat avec les sociologues. Noiriel souligne un point peu abordé jusqu'alors : Simiand se place d'emblée sur le terrain de la philosophie et c'est en philosophe qu'il aborde la critique de l'école méthodique. Seignobos, quant à lui, n'a pas acquis, dans son parcours

118. « Tentons l'expérience », 1989, p. 1322.

119. « Tentons l'expérience », 1989, p. 1322.

120. LEPETIT, 1999, p. 303.

121. « Tentons l'expérience », 1989, p. 1323.

122. «Tentons l'expérience », 1989, p. 1323.

123. Noiriel, 1989. Le titre (« Pour une approche subjectiviste du social ») n'est pas de Noiriel, comme il le précisera par la suite (NoIRIEL, 1990, p. 85).

124. NoIRIEL, 1989, p. 1435.

125. NoIRIEL, 1989 , p. 1438. 
d'historien, « la culture et la maîtrise théoriques requises $[\ldots]^{126}$ » pour engager le débat. L'article de Simiand, remarque Noiriel, vise surtout à fournir une définition légitime du « social », qui est une question politique et scientifique centrale au début du $\mathrm{XX}^{\mathrm{e}}$ siècle. Les sociologues durkheimiens, institutionnellement marginaux, cherchent à imposer leur définition du social par l'imposition de leur méthode (i.e. théoricisme, recherche des invariants, formulation de lois, etc.). Noiriel constate que les désaccords entre les deux disciplines persistent tout au long $\mathrm{du} \mathrm{xx}^{\mathrm{e}}$ siècle, malgré de multiples appels au dialogue. À l'aune de cette opposition - solidifiée et structurelle - entre l'histoire et la sociologie, il propose de réexaminer le lieu commun d'une école des Annales qui aurait mis en œuvre le programme de Durkheim.

La démarche de Noiriel rejoint les propositions faites dix ans plus tôt par Revel dans son article sur les paradigmes des Annales. Toutefois, Noiriel creuse plus avant le dévoilement de l'allant de soi qui fait des historiens des Annales les continuateurs du projet sociologique du début du siècle. Le travail de Febvre est à cet égard un point d'entrée édifiant: Noiriel oppose ainsi l'écriture exubérante, l'empirisme et la délectation monographique de l'historien aux abstractions du sociologue. La démarche durkheimienne part de la question et aboutit au document, alors que les Annales font exactement le chemin inverse ${ }^{127}$. Dans cette perspective, la monographie, parce qu'elle justifie une maîtrise du corpus archivistique, se donne comme l'exercice privilégié des annalistes, encore soumis à une «tyrannie de l'archive » qui les rapproche paradoxalement de Seignobos ${ }^{128}$. Or à l'inverse, la monographie, parce qu'elle n'évacue pas le désir (illusoire) d'une exhaustivité qui empêche de se consacrer aux seules « relations signifiantes ${ }^{129} »$, est nulle et non avenue pour Simiand.

Noiriel interroge également le clivage entre Simiand et Febvre à la lumière du concept de Zusammenhang hérité de Whilelm Dilthey. Febvre cherche à comprendre, à partir des traces, la cohérence d'une époque. Il s'inscrit pleinement dans le paradigme « subjectiviste » de l'historiographie française. Simiand et la sociologie durkheimienne ont à l'inverse rompu avec le « subjectivisme herméneutique » pour acquérir leur autonomie $^{130}$. En examinant a posteriori la frontière qui aurait toujours séparé les Annales de la sociologie (de Simiand), par-delà les discours d'intention qui l'ont masquée, Noiriel entend aussi refonder la légitimité d'une " histoire sociale à part entière ", inscrite dans une tradition Bloch-Febvre renouvelée, simultanément capable d'inventer de « nouvelles formes de dialogue entre l'histoire et la sociologie ${ }^{131} »$. Dans cette perspective, l'interdisciplinarité n'a constitué jusque-là qu'un mirage ${ }^{132}$, voire une aporie, en ce sens qu'elle est restée formulée en termes exclusivement disciplinaires, ce qui la rend inintelligible au-delà de son espace d'énonciation. Le travail de réappropriation de la frontière entre l'histoire défendue par les Annales et la sociologie puise une lecture critique des allants de soi disciplinaires (i.e. Bloch et Febvre réalisant le programme

126. NoIRIEL, 1989, p. 1437.

127. NoIRIEL, 1989, p. 1441.

128. NoIRIEL, 1989, p. 1442.

129. NoIRIEL, 1989, p. 1441.

130. NoIRIEL, 1989, p. 1442-1443.

131. NoIRIEL, 1989, p. 1449.

132. NoIRIEL, 1989, p. 1444. 
de Durkheim, le texte de Simiand comme boussole heuristique) et la formulation d'une série de propositions conceptuelles et pratiques qui rejoindrait le paradigme subjectiviste emprunté en son temps par Lucien Febvre.

Noiriel prend également acte de l'incommensurabilité de l'histoire et de la sociologie et suggère que le dialogue entre historiens et sociologues devienne en fait une " traduction d'un langage à un autre ${ }^{133}$ ». La nécessité d'une traduction des concepts, des méthodes et des approches d'une discipline à l'autre signale, en creux, la réaffirmation disciplinaire esquissée par Revel en 1979 et sous-tendu par l'éditorial des Annales de 1989. Cependant, l'histoire sociale et le paradigme subjectiviste de Noiriel ne peuvent se limiter à quelques emprunts d'innovations sociologiques: il s'agit donc d'" élargir les problématiques [...] à l'ensemble des questions traitées par la sociologie ${ }^{134} »$. En pariant sur « l'aspect cumulatif ${ }^{135} »$ du travail historien, Noiriel propose de reprendre, à nouveaux frais, des objets d'analyse délaissés par l'histoire quantitative. Il suggère ensuite l'emploi de trois concepts oubliés capables de faire naître « de nouveaux champs de recherche, de nouvelles interrogations et de nouvelles explications $[\ldots]^{136} »$. Le premier est l'« expérience vécue », essentiel chez Max Weber et central chez Maurice Halbwachs mais absent des questionnements classiques d'histoire sociale en raison de l'ambiguïté, dénoncée par Simiand dans l'œuvre de Seignobos, de la notion d' " intentions de l'auteur ${ }^{137}$ ». Le second renvoie à l'analyse des processus d'objectivation et de fabrication des catégories. En informant les modes de constitution des évidences catégorielles, il est possible, selon Noiriel, de déconstruire les évidences de l'histoire quantitative. Enfin, le troisième concept mobilisé est celui d'intériorisation (i.e. «l'équivalent, au niveau des individus, du concept d'objectivation pour le monde matériel ») créé par Émile Durkheim et renouvelé par Norbert Elias.

Ainsi, l'article de Noiriel poursuit, approfondit et étend le travail de réappropriation de la frontière avec la sociologie qu'avait entrepris Revel une décennie plus tôt. L'environnement cognitif et institutionnel moins favorable (i.e. hégémonie contestée à l'intérieur de la discipline historique et à l'extérieur) incite les Annales à faire revivre un clivage abandonné par Braudel. Cette redécouverte d'un tracé irréductible entre l'histoire telle que la défendent les Annales et la sociologie passe par une historicisation critique et précise des origines de cette frontière: Bloch et Febvre ont bien cheminé avec la sociologie durkheimienne mais ils n'ont pas, dans leur revue, accompli le programme de Simiand. C'est en revenant à l'origine de ce mythe fondateur que Noiriel fournit aux historiens les instruments d'une démarcation, les outils nécessaires à la reconstruction d'une frontière qui n'a jamais cessé d'être présente. Dès lors, la réactivation de la frontière, par un retour sur des origines chimériques et jamais questionnées, apparaît comme un moyen de légitimer une pratique ouverte (i.e. la traduction, l'emploi de concepts marqués par leurs origines sociologiques) sans céder à un libre-échangisme cognitif échevelé, probablement mortifère dans un moment de recomposition disciplinaire en sciences sociales.

133. NoIRIEL, 1989, p. 1450.

134. NoIRIEL, 1989, p. 1451.

135. NoIRIEL, 1989, p. 1451.

136. NoIRIEL, 1989, p. 1452.

137. NoIRIEL, 1989, p. 1452. 
Les contributions de Revel et Noiriel illustrent une posture horographique qui se démarque des usages braudéliens. Si l'ambition fondationnelle est toujours présente chez Noiriel - l'émergence d'une « socio-histoire » en est l'indice -, la propension à cartographier une fois pour toutes l'espace disciplinaire est mise en suspens. La réflexivité - et la rhétorique qui lui est afférente - est désormais l'arrière-plan des pratiques de démarcation. Les rapports entre histoire et sociologie, qui passent par l'interface des Annales, sont envisagés en référence à l'histoire des usages successifs de l'article fantomatique et rémanent de Simiand. Les contributeurs ne manquent jamais de prendre de la hauteur à travers leurs esquisses de généalogies. Ainsi Antoine Prost dans sa défense de Seignobos - transportée dans la revue Mil neuf cent -, injustement " exécuté » par les durkheimiens et leurs complices en histoire ${ }^{138}$. Le travail de réajustement des positions épistémologiques opère sous la forme d'un déplacement et d'une mise à distance permis par l'histoire des idées et des controverses scientifiques. On refait alors l'histoire sans prétendre y contribuer, on s'autorise d'un point de vue énoncé à partir de nulle part: spectateur plus qu'acteur. La réflexivité manifestée dans les discours des historiens n'est pas sans évoquer ici la réflexivité à l'œuvre dans la disputatio philosophique telle que l'analyse Jean-Louis Fabiani: « [...] la condition principale de la réflexivité philosophique considérée en général réside dans la réflexivité même de la dispute, qui se prend elle-même pour objet et qui investit ses propres mécanismes ${ }^{139}$. " Toutefois, chaque contributeur n'en cède pas moins à une forme d'identification projective, en ce sens qu'il attribue indéfectiblement à l'une ou l'autre des positions intellectuelles générées par le débat de 1903 des significations positives ou négatives (selon les cas) et qu'il reconnaît comme pertinentes. Ce faisant, les chercheurs y trouvent aussi l'instrument d'une auto-analyse. En témoigne la constance avec laquelle Revel s'en saisit et investit sur cette histoire (récemment en 2007), actualisant dans chaque nouvel article ses propres conjectures. Enfin, le retour (et le recours) à Simiand est largement ritualisé. Le recours à l'article-fétiche de 1903 marque la fabrique de l'identité disciplinaire de l'histoire. Et ce n'est pas un hasard s'il réapparaît presque toujours dans les Annales à l'occasion de commémorations et de moments de crise. Dans la biographie collective de l'histoire des Annales aventurées en terra sociologica, il est un point d'ancrage aussi déterminant qu'il est sous-terrain.

\section{EFFETS DE SEUILS ET TOPIQUES DE L'ALTÉRITÉ}

Cerner le positionnement différentiel d'une revue par rapport à une discipline nous a tout d'abord permis de mesurer, peut-être de façon suraiguë, les évolutions et les déplacements du paysage des sciences humaines et sociales. Les Annales se sont d'abord installées (volontairement et presque artificiellement) à la marge de la discipline de l'histoire, puis n'ont cessé d'accroître leur influence au point de se désigner à partir des années 1960 puis d'apparaître comme la quasi-incarnation éditoriale de toute la discipline. Les trois configurations horographiques choisies - composant autant

138. Prost, 1994.

139. FABIANI, 2004. 
d'événements discursifs - ont permis d'envisager la frontière entre les Annales et la sociologie comme un rapport de force, c'est-à-dire une dynamique de structuration et de mise en cohérence des écarts et des convergences épistémologiques ébauchée par les Annales. C'est un rapport de force très complexe puisqu'il intègre également une dynamique institutionnelle qui tient compte de la place qu'occupe chacun des acteurs dans l'ordre universitaire. Les positions épistémologiques et institutionnelles constituent le point de départ des tensions.

Un jeu (au sens de règles permettant un nombre de mouvements et de déplacements finis) est engagé qui définit des configurations possibles de la délimitation, des configurations conditionnées par un ensemble de contraintes (position de la discipline ou de la revue dans l'ordre académique, degré d'influence dans l'espace universitaire, emprise épistémologique). Dans la confrontation entre les Annales et la sociologie, il est remarquable que les acteurs en présence travaillent la (pensée de la) frontière sans remettre en question les règles du jeu (contestation d'un positionnement institutionnel, refus de prendre en compte les arguments cognitifs de l'adversaire). Les entités disciplinaires (et ce qu'elles peuvent représenter ou évoquer pour les acteurs) sont investies dans les argumentations cartographiques. Les zones cognitives sont quadrillées à l'aide de ces registres discursifs génériques, le plus souvent à la limite du stéréotype. La réappropriation de l'histoire des frontières permet aussi un dévoilement des mythes originels. Le procès de légitimation dans lequel s'engagent les Annales dans les années 1980 passe précisément par une remise en question des évidences, des allants de soi et des poncifs. Revel puis Noiriel réactivent, par l'histoire, un clivage que des circonstances plus favorables avaient conduit à ignorer ou plutôt à oublier. La frontière est ici introduite en territoire-refuge, en espace-protégé; son historicisation l'inscrit dans la permanence et son éventuel franchissement est davantage maîtrisé.

La frontière ne doit pas seulement être envisagée comme un rapport à l'extérieur. Les prises de position différentes de Bloch et Febvre à l'endroit de la sociologie invitent à penser la frontière en termes de conflit interne. Les effets concrets et perceptibles d'un déplacement de frontière ne sont pas (dans le cas des Annales et de la sociologie) le produit d'une rhétorique visant à redéfinir les points d'incompatibilité et les convergences. Ce travail discursif sert d'abord à matérialiser le rapport de force, à le rendre visible - quitte à surjouer le jeu des oppositions disciplinaires. Il indique également une certaine forme d'altérité (i.e. compréhension et discussion des problématiques extérieures à la revue, au groupe de travail ou à la discipline). Saisie à la fois comme rapport de force, tension interne et signe d'altérité, la frontière apparaît alors comme un enjeu en ce sens qu'elle condense l'ensemble des dimensions stratégiques, institutionnelles, épistémologiques et cognitives qui permet à une communauté d'acteurs (rassemblés dans des cadres aux formes variées, i.e. revue, discipline dans le cas étudié ici) de se reconnaître entre eux et de se définir. La frontière laisse apparaître un noyau de valeurs et de références (heuristiques, langagières, organisationnelles) partagées (jusque dans leur discussion et leur remise en cause) par un collectif dont les membres peuvent ensuite se saisir pour l'approuver, la dénoncer, la transformer, dans un mouvement continuel d'agencement et d'ajustement.

Notre travail n'épuise pas la complexité des phénomènes étudiés ni ne se soustrait à ces logiques. Il y participe pleinement même. Paraissant dans la Revue de synthèse 
plus d'un siècle après l'article de Simiand, il met à l'épreuve une forme (ou un registre) de réflexivité. On retrouve cette disposition interprétative dans les essais de Revel, à ceci près que l'attention portée sur ce que nous avons nommé l'horographie a amené à formuler de nouvelles questions. Ainsi, nous avons pris acte de l'omniprésence du discours sur les frontières. La frontière, et plus généralement l'ensemble des motspivots ressortissant de la métaphore spatiale, frappent par leur ubiquité. Il n'est pas d'argumentation qui, à un moment ou à un autre, ne s'y rapporte ${ }^{140}$. En sorte que la frontière est pour notre analyse ce que la discontinuité représente pour l'archéologie foucaldienne: une notion paradoxale, «puisqu'elle délimite le champ dont elle est l'effet; puisqu'elle permet d'individualiser les domaines, mais qu'on ne peut l'établir que par comparaison ${ }^{141} »$. Le terrain glissant de l'enquête paraît alors se dérober à l'enquête horo-génétique. Mais c'est justement ce vers quoi elle veut tendre. La déconstruction des dynamiques par lesquelles des acteurs établissent l'unité et la singularité relatives d'ensembles épistémologiques et institutionnels dévoile les allants de soi, les attendus et les refoulés de l'imaginaire des frontières.

\section{LISTE DES RÉFÉRENCES}

Аввотт (Andrew), 1995, "Things of Boundaries", Social Research, vol. LXII, ${ }^{\circ} 4$, p. $857-882$.

Аввотт (А.), 1999, Department and Discipline. Chicago Sociology at One Hundred, Chicago, Chicago University Press.

Аввотт (A.), 2001, Times Matters. On Theory and Methods, Chicago, Chicago University Press.

Aymard (Maurice), 1988, « Histoire et sociologie », dans Mendras (Henri) et Verret (Michel), éd., Les Champs de la sociologie française, Paris, Armand Colin, p. 221-232.

Berthelot (Jean-Michel), 1998, « Histoire et sociologie: une affaire de discipline », Recherches sociologiques, vol. XXIX, $\mathrm{n}^{\circ}$ 3, p. 23-43.

Besnard (Philippe), 1979, «La formation de l'équipe de l'Année sociologique », Revue française de sociologie, vol. $\mathrm{XX}, \mathrm{n}^{\circ} 20$, fasc. 1, p. 7-31.

Besnard (P.), 2003, Études durkheimiennes, Genève, Droz.

B sociale, vol. III, p. 590-592.

Bцoch (M.), 1934, «Histoire, doctrine économique, sociologique », Annales d'histoire économique et sociale, vol. VI, p. 510.

Bloch (M.), 1935, «Les Annales sociologiques », Annales d'histoire économique et sociale, vol. VII, p. 393.

Bцoch (M.), 1936, «La sociologie et le passé du droit», Annales d'histoire économique et sociale, vol. VIII, p. 458.

Bourdieu (Pierre), 1995, « Sur les rapports entre la sociologie et l'histoire en Allemagne et en France », Actes de la recherche en sciences sociales, vol. CVI, n 1 , p. 108-122.

140. Le phénomène s'étend à la formation de paradigmes sociologiques syncrétiques et intégrateurs, comme l'a bien signalé SILBER, 1995.

141. Foucault, 1969, p. 17. 
Braudel (Fernand), 1953, « Georges Gurvitch et la discontinuité du social », Annales. Économies, sociétés, civilisations, $\mathrm{n}^{\circ}$ 3, p. 347-361.

Braudel (F.), 1958, «Histoire et sciences sociales: la longue durée », Annales. Économies, sociétés, civilisations, $\mathrm{n}^{\circ} 4$, p. 725-753.

Braudel (F.), 1969, Écrits sur l'histoire, Paris, Flammarion.

Braudel (F.), 1997, Les Ambitions de l'histoire, Paris, Éditions de Fallois.

Burguière (André), 1979, « Histoire d'une histoire: la naissance des Annales », Annales. Économies, sociétés, civilisations, $\mathrm{n}^{\circ}$ 6, p. 1347-1359.

Burguière (A.), 2006, L'École des Annales. Une histoire intellectuelle, Paris, Odile Jacob.

Cefaï (Daniel), 2007, Pourquoi se mobilise-t-on? Les théories de l'action collective, Paris, La Découverte.

Chartier (Roger) et Revel (Jacques), 1979, «Lucien Febvre et les sciences sociales », Historiens et géographes, $\mathrm{n}^{\circ} 272$, p. 427-442.

CraIg (John), 1979, «Maurice Halbwachs à Strasbourg », Revue française de sociologie, vol. XX, n ${ }^{\circ}$, p. $273-292$.

Craig (J.), 1983, «Sociology and Related Disciplines Between the Wars. Maurice Halbwachs and the Imperialism of the Durkheimians », dans BESNARD (Philippe), éd., The Sociological Domain. The Durkheimians and the Founding of the French Sociology, Cambridge/Paris, Cambridge University Press/Éditions de la Maison des sciences de l'Homme, p. 263-289.

CraIg (J.), 1984, Scholarship and Nation Building. The Universities of Strasbourg and Alsatian Society, 1870-1939, Chicago, University of Chicago Press.

DaIX (Pierre), 1995, Braudel, Paris, Flammarion.

Delacroix (Christian), Dosse (François) et Garcia (Patrick), 2007, Les Courants historiques en France $X I X^{e}-X X^{e}$ siècle, Paris, Gallimard.

Dosse (François), 1987, L'Histoire en miettes. Des « Annales » à la «nouvelle histoire », Paris, La Découverte.

DuRKheIm (Émile), 1888, « Leçon d'ouverture du cours de sciences sociales », Revue internationale de l'enseignement, vol. XV, p. 23-48.

DuRkheim (É.), 1897, «Préface », L'Année sociologique, vol. I, p. III.

Evans (Robert), 2005, «Demarcation Socialized. Constructing Boundaries and Recognizing Difference ", Science, Technology and Human Values, vol. XXX, n 1, p. 3-16.

Fabiani (Jean-Louis), 1988, Les Philosophes de la république, Paris, Éditions de Minuit.

FABIANI (J.-L.), 2003, « Pour en finir avec la réalité unilinéaire. Le parcours méthodologique de Andrew Abbott ", Annales. Histoire, sciences sociales, n 3, p. 549-565.

FABIANI (J.-L.), 2004, « La sociologie historique face à l'archéologie du savoir », dans Foucault: usages et actualités, $\mathrm{n}^{\circ}$ spéc. de la revue Le Portique, $\mathrm{n}^{\circ}$ 13-14 (édition en ligne).

FABiAnI (J.-L.), 2006, «À quoi sert la notion de discipline? », dans Boutier (Jean), Passeron (Jean-Claude) et Revel (Jacques), Qu'est-ce qu'une discipline?, Paris, Éditions de l'École des hautes études en sciences sociales, p. 11-34.

FABIANI (J.-L.), 2007, « Disputes, polémiques et controverses dans les mondes intellectuels. Vers une sociologie historique des formes de débat agonistique », Mil neuf cent, vol. XXV, $\mathrm{n}^{\circ} 1$, p. $45-60$.

Febvre (Lucien), 1930, «Histoire, économie et statistique », Annales d'histoire économique et sociale, vol. II, $\mathrm{n}^{\circ}$ 8, p. 581-590.

Febvre (L.), 1933, «Pour les historiens, un livre de chevet», Annales d'histoire économique et sociale, vol. $\mathrm{V}, \mathrm{n}^{\circ} 20$, p. 161-163.

Febvre (L.), 1941, «La société féodale: une synthèse critique », Annales d'histoire sociale, vol. III, p. 125-130. 
Febvre (L.), 1953, Combats pour l'histoire, Paris, Armand Colin.

Febvre (L.), 1963, Pour une histoire à part entière, Paris, SEVPEN.

Foucault (Michel), 1969, L'Archéologie du savoir, Paris, Gallimard.

FouCHER (Michel), 1991, Fronts et frontières. Un tour du monde géopolitique, Paris, Fayard.

FOURNIER (Marcel), 2007, Émile Durkheim. 1858-1917, Paris, Fayard.

Friedman (Susan), 1996, Marc Bloch, Sociology and Geography. Encountering Changing Disciplines, Cambridge, Cambridge University Press.

Gieryn (Thomas), 1995, « Boundaries of Science », dans JASANOFf (Sheila) et al., éd., Handbook of Science and Technology Studies, Thousand Oaks, Sage, p. 394-443.

Gieryn (T.), 2001, Cultural Boundaries of Science. Credibility on the Line, Chicago, Chicago University Press.

Gurvitch (Georges), 1957, «Continuité et discontinuité en histoire et en sociologie », Annales. Économies, sociétés, civilisations, $\mathrm{n}^{\circ} 1, \mathrm{p} .73-84$.

Gurvitch (G.), 1958, « Objet et méthode de la sociologie », dans ID., dir., Traité de sociologie, Paris, Presses universitaires de France, p. 3-27.

GuRvitch (G.), 1962, Dialectique et sociologie, Paris, Flammarion.

Halbwachs (Maurice), 1936, «La méthodologie de François Simiand. Un empiriste rationaliste », Revue philosophique, vol. CXXI, p. 281-319.

Hall (John), 1999, Cultures of Inquiry. From Epistemology to Discourse in Sociohistorical Research, Cambridge, Cambridge University Press.

HeIlbron (Johan), 1985, « Les métamorphoses du durkheimisme, 1920-1940 », Revue française de sociologie, vol. XXVI, $\mathrm{n}^{\circ}$ 2, p. 203-237.

HeXTer (Jack), 1972, «Braudel and the monde braudélien», Journal of Modern History, vol. XLIV, $n^{\circ} 4$, p. 480-539.

«Histoire et sciences sociales. Un tournant critique?», 1988, Annales. Économies, sociétés, civilisations, $\mathrm{n}^{\circ} 2$, p. 291-293.

Hourmant (François), 2007, « De la double nature des revues et des ruptures », dans Baudouin (Jean) et Hourmant (François), dir., Les Revues et la dynamique des ruptures, Rennes, Presses universitaires de Rennes, p. 11-20.

KARADY (Victor), 1976, «Durkheim, les sciences sociales et l'Université: bilan d'un semiéchec », Revue française de sociologie, vol. XVII, n² 2, p. 267-311.

Karady (V.), 1979, « Stratégies de réussite et modes de faire-valoir de la sociologie chez les durkheimiens ", Revue française de sociologie, vol. XX, p. 49-82.

Lamont (Michèle) et Molnar (Virag), 2002, «The Study of Boundaries in the Social Sciences », Annual Review of Sociology, vol. XXVIII, p. 167-195.

LePETIT (Bernard), 1999, Carnet de croquis. Sur la connaissance historique, Paris, Albin Michel.

Leroux (Robert), 1998, Histoire et sociologie en France. De l'histoire-science à la sociologie durkheimienne, Paris, Presses universitaires de France.

«Les Annales, 1929-1979», 1979, Annales. Économies, sociétés, civilisations, n 6, p. 13441346.

Leuillot (Philippe), 1973, « Aux origines des “Annales d'histoire économique et sociale” (1928). Contribution à l'historiographie française ", dans Mélanges en l'honneur de Fernand Braudel, t. II : Méthodologie de l'histoire et des sciences humaines, Toulouse, Privat, p. 317-324.

LÉvi-Strauss (Claude), 1949, " Histoire et ethnologie », Revue de métaphysique et de morale, $\mathrm{n}^{\circ}$ 3-4, p. 363-391.

Maillard (Alain), 2005, «Les temps de l'historien et du sociologue. Retour sur la dispute Braudel-Gurvitch », Cahiers internationaux de sociologie, vol. CXIX, n 2, p. 197-222. 
Mucchielli (Laurent), 1995, « Aux origines de la nouvelle histoire en France. L'évolution intellectuelle et la formation du champ des sciences sociales (1880-1930) », Revue de synthèse, $\mathrm{n}^{\circ} 1$, p. 55-98.

Mucchielli (L.), 1998, La Découverte du social. Naissance de la sociologie en France, Paris, La Découverte.

NoIRIEL (Gérard), 1989, «Pour une approche subjectiviste du social», Annales. Économies, sociétés, civilisations, ${ }^{\circ}$ 6, p. 1435-1459.

Noiriel (G.), 1990, « Naissance du métier d'historien », Genèses, nº 1, p. 58-85.

NoIrIel (G.), 2005, Sur la « crise » de l'histoire, Paris, Gallimard.

Passeron (Jean-Claude), 1991, Le Raisonnement sociologique. Un espace non poppérien de l'argumentation, rééd. Paris, Albin Michel, 2006.

Prochasson (Christophe), 1996, « Histoire et sociologie, Henri Berr et les durkheimiens (19001914) », dans Biard (Agnès), Bourel (Dominique) et Brian (Éric), dir., Henri Berr et la culture du XXe siècle. Histoire, science et philosophie, Paris, Albin Michel, p. 61-79.

Prost (Antoine), 1994, "Charles Seignobos revisité », Vingtième siècle. Revue d'histoire, vol. XLIII, p. 100-118.

Rebérioux (Madeleine), 1983, «Le débat de 1903. Historiens et sociologues », dans CARBonell (Charles-Olivier) et Livet (Georges), éd., Au berceau des Annales. Le milieu strasbourgeois. L'histoire en France au début du XX siècle, Toulouse, Presses de l'Institut d'études politiques, p. 219-230.

Revel (Jacques), 1979, « Histoire et sciences sociales: les paradigmes des Annales », Annales. Économies, sociétés, civilisations, $\mathrm{n}^{\circ}$ 6, p. 1360-1376.

Revel (J.), 1995, «Histoire et sciences sociales: une confrontation instable », dans Boutier (Jean) et Julia (Dominique), éd., Passés recomposés. Champs et chantiers de l'histoire, Paris, Autrement, p. 69-81.

Revel (J.), 1999, « Histoire et sciences sociales. Lectures d'un débat français autour de 1900 », dans Bödeker (Hans Erich), Hanns (Peter), Schlumbohum (Jürgen), éd., Wissenschaft als kulturelle Praxis, 1750-1900, Göttingen, Vandenhoeck \& Ruprecht, p. 377-399.

Revel (J.), 2001, «Les sciences historiques », dans Berthelot (Jean-Michel), dir., Épistémologie des sciences sociales, Paris, Presses universitaires de France, p. 21-76.

Revel (J.), 2007, «Histoire et sciences sociales. Lectures d'un débat français autour de 1900 », Mil neuf cent, vol. XXV, $\mathrm{n}^{\circ} 1, \mathrm{p} .101-126$.

Seignobos (Charles), 1901, La Méthode historique appliquée aux sciences sociales, Paris, Alcan.

Silber (Ilana), 1995, « Space, Fields, Boundaries, the Rise of Spatial Metaphors in Contemporary Sociological Theory », Social Research, vol. LXII, n² 2, p. 323-355.

Simiand (François), 1903, « Méthode historique et science sociale », Revue de synthèse historique, $\mathrm{n}^{\circ} 16$, p. $1-22$, et $\mathrm{n}^{\circ} 17$, p. 129-157; rééd. dans les Annales. Économies, sociétés, civilisations, $\mathrm{n}^{\circ} 1,1960$, p. 83-119.

«Tentons l'expérience », 1989, Annales. Économies, sociétés, civilisations, n 6, p. 1317-1323.

Topalov (Christian), 1997, « Maurice Halbwachs et les villes (1908-1912). Une enquête d'histoire sociale des sciences sociales », Genèse, vol. LII, $\mathrm{n}^{\circ}$ 5, p. 1057-1083.

Topalov (C.), 2004, «Les usages stratégiques de l'histoire des disciplines. Le cas de l'“école de Chicago" en sociologie », dans HeIlbron (Johan), Lenorr (Remi) et SAPIro (Gisèle), Pour une histoire des sciences sociales. Hommage à Pierre Bourdieu, Paris, Fayard, p. 127-157.

"Une correspondance entre Lucien Febvre et François Simiand à l'aube des “Annales" », 1989, Vingtième siècle. Revue d'histoire, $\mathrm{n}^{\circ} 23$, p. 103-110. 